\author{
UNIVERSIDADE DE SÃO PAULO \\ INSTITUTO DE FÍSICA DE SÃO CARLOS
}

EDMILSON ROQUE DOS SANTOS

Discontinuous transitions to collective dynamics in star motifs of coupled oscillators

São Carlos

2018 



\title{
Discontinuous transitions to collective dynamics in star motifs of coupled oscillators
}

\author{
Dissertation presented to the Physics Grad- \\ uate Program at the São Carlos Institute of \\ Physics, University of São Paulo, to obtain \\ the degree of Master of Sciences. \\ Concentration area: Basic Physics \\ Advisor: Prof. Dr. Tiago Pereira
}

Corrected version

(Original version available on the Program Unit)

São Carlos

2018 
I AUTHORIZE THE REPRODUCTION AND DISSEMINATION OF TOTAL OR PARTIAL COPIES OF THIS DOCUMENT, BY CONVENCIONAL OR ELECTRONIC MEDIA FOR STUDY OR RESEARCH PURPOSE, SINCE IT IS REFERENCED.

Cataloguing data revised by the Library and Information Service of the IFSC, with information provided by the author

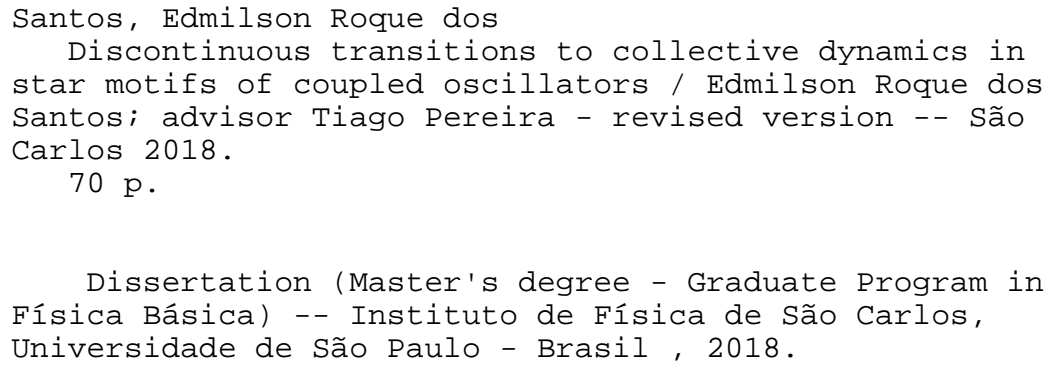

1. Synchronization. 2. Coupled oscillators. 3. Discontinuous transitions. I. Pereira, Tiago, advisor. II. Title. 


\section{Acknowledgements}

I want to thank Jaap Eldering and Tiago Pereira for supervising me in this Master and guiding me in the challenge to write more precisely my ideas. I am in debt with some people that contributed throughout this project: Eric Andrade, Francisco Rodrigues, Fred Brito, Gabriela Depetri, Helcio Martinho, Hildebrando Rodrigues and colleagues from the seminar, Leonardo Maia, Maria Michalska, Partha Guha, Stefan Ruschel and Thomas Peron.

I thank all my other friends which contributed in different manners beyond the academic scenario. A special thanks to Larissa, and last but not least, Edmilson, Gabriela, Rojanira for supporting and bringing me to what I am nowadays.

I acknowledge CAPES for the financial support. 

“(..)

Ed il mio bacio scioglierà

il silenzio che ti fa mia!

Tramontate, stelle!

All'alba vincerò!

Vincerò! Vincerò!"

(Nessun Dorma - Giacomo Puccini)

"It's not who I am underneath, but what I do defines me."

(Batman Begins) 



\section{Abstract}

SANTOS, E. R. Discontinuous transitions to collective dynamics in star motifs of coupled oscillators. 2018. 70 p. (Mestrado em Ciências) - Instituto de Física de São Carlos, Universidade de São Paulo, São Carlos, 2018.

This dissertation is dedicated to the rigorous study of discontinuous transitions in star graphs of coupled phase oscillators. A star graph consists of a central node, called hub, connected to peripheral nodes called leaves. We consider the setting where the frequency of the leaves is identical and the hub has a higher frequency when isolated. This captures the effect of positive correlation between the hub high number of connections and its high natural frequency. Hub higher frequency turns out to be the key feature for discontinuity in the transition from incoherent to synchronous behavior. This transition has been observed numerically and explained via a non-rigorous analytical treatment in the thermodynamic limit. Using Möbius group reduction and the theory of persistence of normally hyperbolic invariant manifold, we prove that this transition is indeed discontinuous for a certain set of initial conditions.

Keywords: Synchronization. Coupled oscillators. Discontinuous transitions. 



\section{Resumo}

SANTOS, E. R. Transições descontínuas para dinâmica coletiva em estruturas de estrelas de osciladores acoplados. 2018. 70 p. Dissertação (Mestrado em Ciências) - Instituto de Física de São Carlos, Universidade de São Paulo, São Carlos, 2018.

Esta dissertação dedica-se em estudar rigorosamente transições descontínuas de osciladores de fase acoplados em grafos estrelas. Um grafo estrela é composto de um nó central, chamado hub, conectado a nós periféricos chamados folhas. Consideramos a situação na qual a frequência das folhas é igual e o hub tem frequência mais elevada, o efeito de correlação positiva entre o grande número de conexões do hub e sua frequência. A elevada frequência do $h u b$ resulta por ser o aspecto crucial na descontinuidade da transição do comportamento incoerente para o síncrono. Esta transição foi observada numericamente e estudada por meio de tratamento analítico não rigoroso no limite termodinâmico. Usando técnica de redução a partir do grupo de Möbius e a teoria de variedades invariantes normalmente hiperbólicias, provamos que esta transição é de fato descontínua para certo conjunto de condições iniciais.

Palavras-chave: Sincronização. Osciladores acoplados. Transições descontínuas. 



\section{List of Figures}

Figure 1 - Star graph: central node in blue represents a hub and in orange are the leaves. . . . . . . . . . . . . . . . . 20

Figure 2 - Four-distinct points define unique circle passing through them and a Möbius transformation which maps $z$ 's to w's. Cross-ratio of both set of points is equal. . . . . . . . . . . . . . . . . 32

Figure 3 - Stable and unstable linear invariant subspaces $E^{s}$ and $E^{u}$ respectively. $W_{l o c}^{s}\left(x_{0}\right)$ and $W_{l o c}^{u}\left(x_{0}\right)$ represent nonlinear invariant manifold tangent to respective linear spaces at fixed point $x_{0} \ldots \ldots \ldots$. . . . . . . . 43

Figure 4 - Two function $f$ and $g$ are $C^{0}$-close. In the plot $f(x)$ has a unique fixed point at $x_{0}=0$. On the other hand, $g(x)$ possesses couple fixed points,

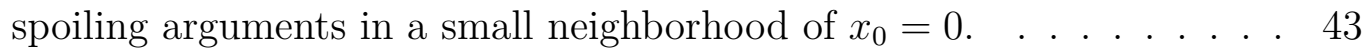

Figure 5 - After a perturbation the system got two new stable and unstable nonlinear invariant manifolds, $\tilde{W}_{l o c}^{s}\left(\tilde{x}_{0}\right)$ and $\tilde{W}_{l o c}^{u}\left(\tilde{x}_{0}\right)$ respectively, at $\tilde{x}_{0}$ which is the perturbed fixed point. Unperturbed invariant manifolds are indicated in dashed-lines. Note that outside neighborhood $V$, they do not coincide or are close anymore. . . . . . . . . . . . . . . . . 44

Figure 6 - Normal hyperbolicity representation: (a) $M$ is a normally attracting invariant manifold, where there are contracting transverse directions represented in orange dashed lines. Blue solid line consists of a trajectory starting in $x$ and evolved by the flow $\varphi^{t}$. After solution enters $M$, remains there for all time. (b) $\tilde{M}$ is the perturbed version of $M$. The trajectory and the perturbed manifold keep their qualitative properties though wiggled a bit. In contrast, prior structures are dashed-displayed. 46

Figure 7 - Order parameter measures phase synchronization. The asynchronous state, identified by the order parameter $|r| \ll 1$, is represented in the left situation. The right plot presents the synchronous state where order

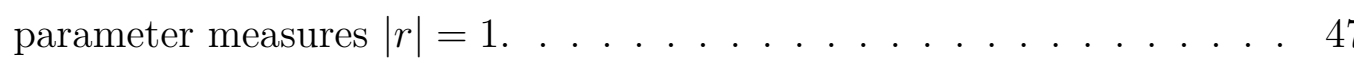

Figure $8-$ Geometrical interpretation of vectors $\mathbf{v}$ and $\mathbf{w}$. The fixed point $\zeta-v$ is arbitrary shown as a unit vector. Since the intern product between $\mathbf{v}$ and unit vector with $\zeta-v$ is negative, they are more the $\pi / 2$ apart. 



\section{List of Abbreviations and Acronyms}

NHIM Normally Hyperbolic Invariant Manifold 



\section{List of Symbols}

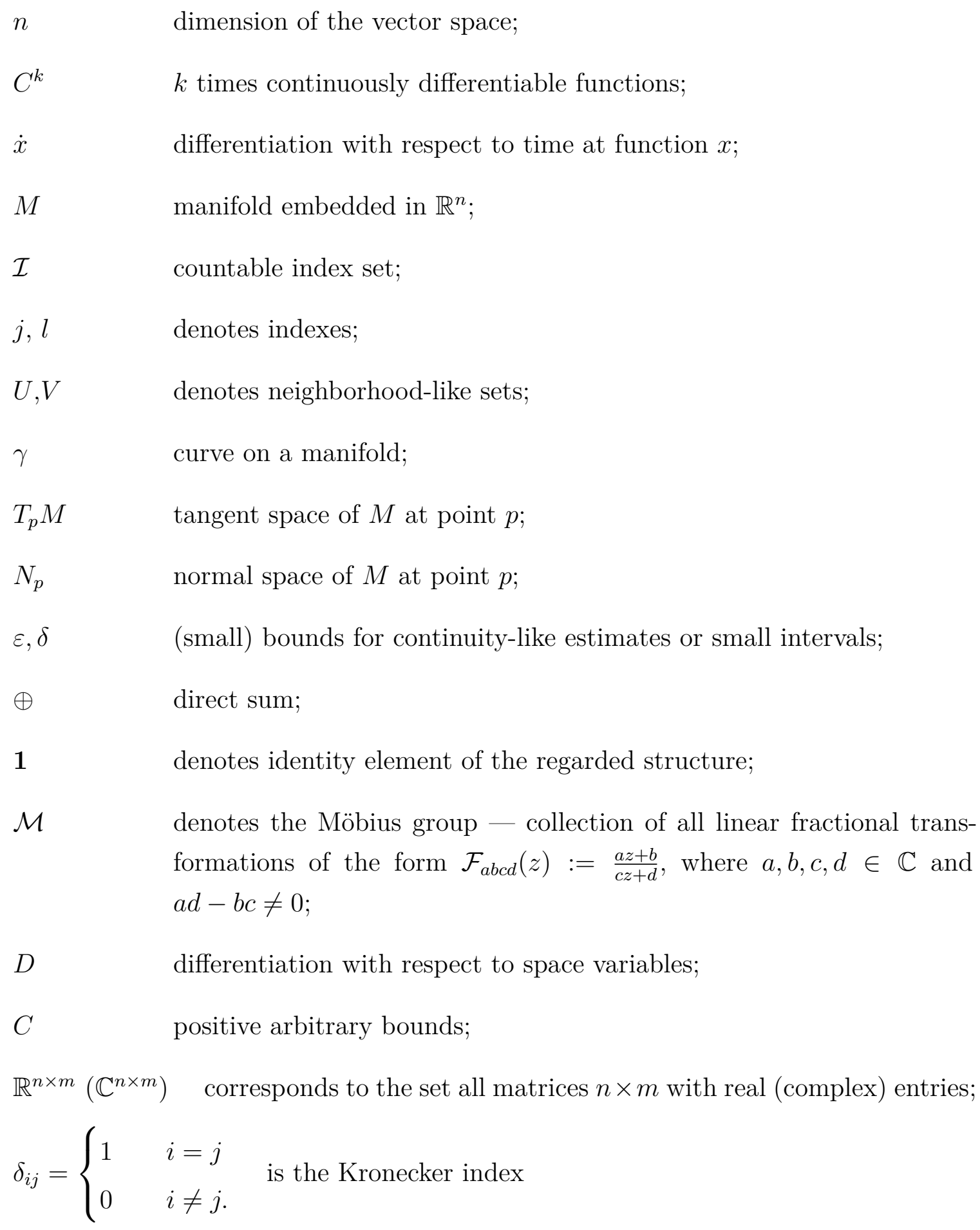





\section{Contents}

$1 \quad$ INTRODUCTION $\ldots \ldots \ldots \ldots \ldots \ldots$

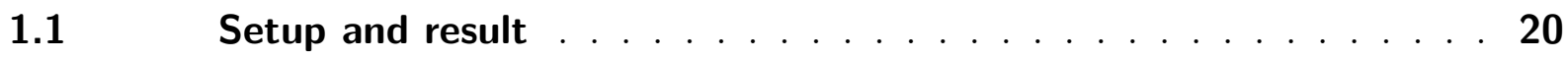

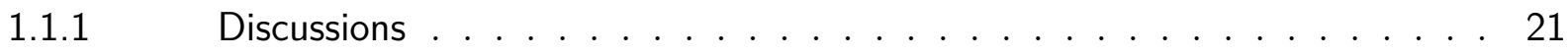

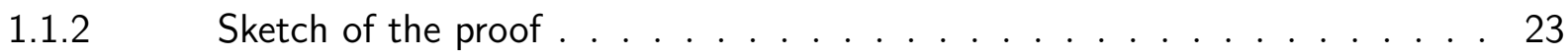

2 LIE GROUPS AND PROJECTIVE GEOMETRY . . . . . . . 25

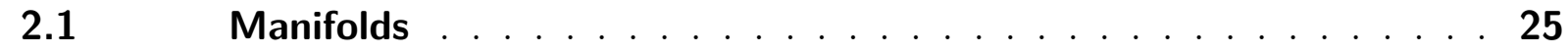

2.2 Lie Groups and Möbius transformation . . . . . . . . . . . 29

$2.2 .1 \quad$ Möbius group . . . . . . . . . . . . . . . . . 30

$2.3 \quad$ Lie Theorem $\ldots \ldots \ldots \ldots \ldots \ldots$

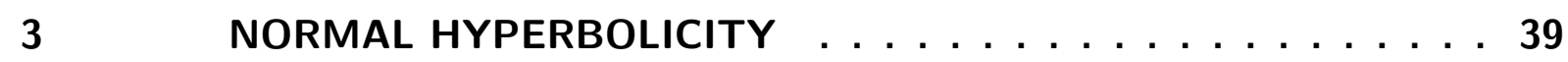

$3.1 \quad$ Characterizing macroscopic states $\ldots \ldots \ldots \ldots$

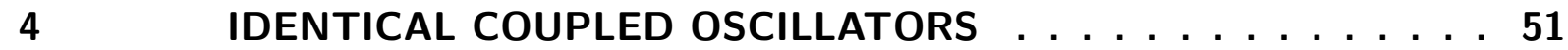

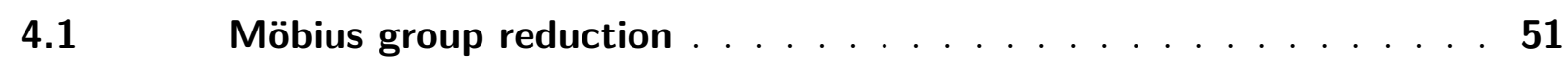

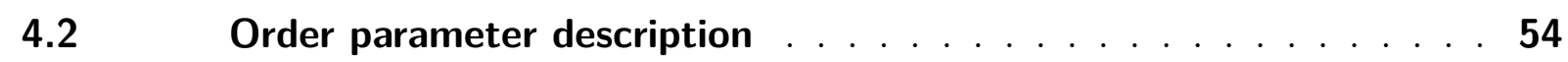

5 STATEMENT OF THE MAIN THEOREM AND PROOF . . . . . 57

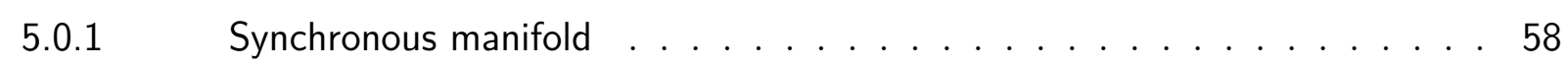

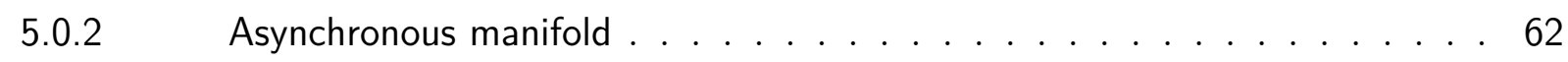

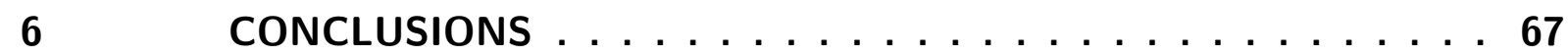

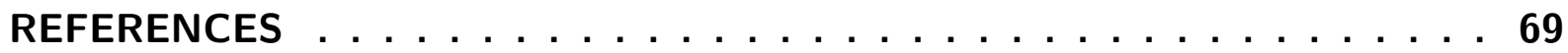





\section{Introduction}

In 2011 Gómez-Gardeñes et al. (1) performed numerical experiments on phase oscillators coupled in a scale-free network possessing a distinct feature: the isolated frequency of each oscillator was the same as its number of connections. The correlation scheme between the network structure and the dynamics led to interesting overall dynamical behavior. They found out that the system's level of synchrony undergoes an apparent discontinuous transition when the coupling strength among oscillator was increased. The authors named this transition explosive synchronization because of the abrupt nature and also evidence of a hysteresis phenomenon.

Starting from an incoherent state, the coupling was increased and after it crossed a critical coupling, the so-called forward critical coupling, all of sudden the network exhibited a high level of synchrony, a synchronous state. On the other hand, for decreasing coupling the network displays an abrupt transition back to incoherent dynamics past a critical value called backward critical coupling. Both critical couplings were distinct, unveiling the hysteresis.

The scaling law for the hysteresis region were obtained heuristically by Zou et al. (2) where they showed that the correlation scheme induced a distinct basin of attraction between the incoherent and coherent states. Futhermore Skardal, Taylor and Sun (3) suggested numerically that a match between heterogeneity of frequencies and network structure (positively correlated) is a key ingredient for optimizing synchronization - consisting in the similar hypothesis used primarily by Gómez-Gardeñes et al.(1) Explosive synchronization has been observed in experimental setup and generalized to time-delay coupling.(4)

The conjecture on the discontinuous transition was also investigated via meanfield approximations in the continuum limit. Peron and Rodrigues (5) and Coutinho and co-authors (6) analyzed explosive synchronization on scale-free networks and obtained excellent analytical prediction corroborated by numerical experiments. From a mathematical perspective such mean-field approach is challenging as it assumes continuity of the order parameter transition for varying coupling, which is not the case for the phenomenon under study.

In 2015 Vlasov and co-authors (7) analyzed such transition in the thermodynamic limit based on Möbius group reduction technique, predicting the transition correctly without need for a mean-field approximation. They studied a star graph consisting of a central highly connected node, called $h u b$, and $N$ peripheral nodes, called leaves, linked to the hub - see Figure 1. Star graph was chosen because it serves as a motif.(8) The authors did 


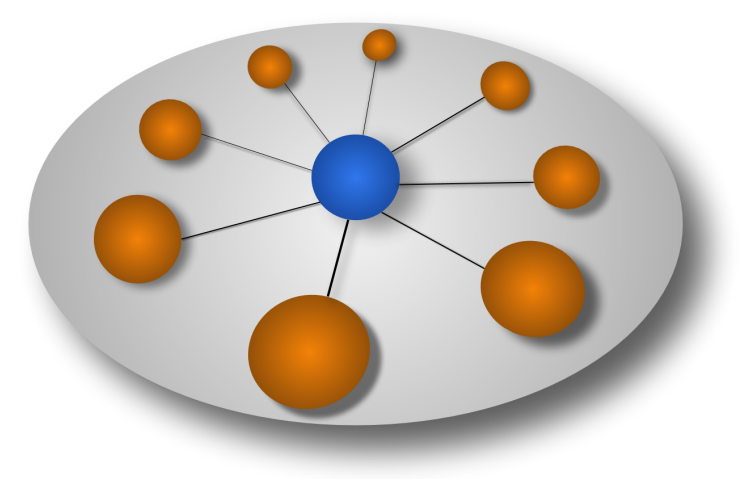

Figure 1 - Star graph: central node in blue represents a hub and in orange are the leaves. Source: By the author.

not, however, set this scenario in rigor and this will be the main aim of this dissertation.

Last year Eldering and collaborators proved results involving the connection between explosive synchronization and chimera state in coupled star graphs (their paper will be published soon). Their main contribution was showing that the incoherent state is the dynamics on a normally hyperbolic invariant manifold for any finite size star. Therefore, we addressed to prove rigorously that the transition phenomenon from incoherent to coherent state is discontinuous and provide a complete exposition of their results to the case of a single star.

\subsection{Setup and result}

Consider a star system (1.1) with $N$ leaves coupled with the hub

$$
\begin{aligned}
& \dot{\vartheta}_{k}=\omega+K \sin \left(\vartheta_{H}-\vartheta_{k}-v\right) \\
& \dot{\vartheta}_{H}=\beta \omega+\beta \frac{K}{N} \sum_{j=1}^{N} \sin \left(\vartheta_{j}-\vartheta_{H}-v\right),
\end{aligned}
$$

where $\vartheta_{k} \in S^{1}$ is the phase of the $k$-th leaf, $\vartheta_{H} \in S^{1}$ is the phase of the hub, $\omega \in \mathbb{R}$ is the natural frequency, $\beta \in \mathbb{R}$ is the correlation parameter, $K$ is the coupling strength and $v \in S^{1}$ is a phase shift.

The parameter $\beta \in(1, \infty)$ quantifies the positive correlation between the hub's natural frequency and its number of connections. For $\beta \in(0,1)$ represents the opposite case, negative correlation, and the system always reaches synchronous state for any positive coupling. We will not analyze this case since there is no discontinuous transition or hysteresis phenomenon. 
Result. When the initial condition for the phases is almost the uniform distribution on unit circle, the system behaves incoherently. The gradual increase of the coupling strength alters this macroscopic state to coherent behavior abruptly after passing over a threshold coupling, so-called forward critical coupling $K_{c}^{f}$. On the other hand, if the phase are chosen to be in the synchronous state and one decreases slowly the coupling, then after a critical value named backward critical coupling $K_{c}^{b}$, incoherent state is achieved again suddenly.

In order to capture the collective properties, we describe them by the order parameter $r: \mathbb{T}^{N+1} \rightarrow \mathbb{C}$. Denote a solution curve of the system (1.1) by $\gamma: \mathbb{R} \rightarrow \mathbb{T}^{N+1}$ and order parameter given by

$$
r(\gamma(t))=\frac{1}{N} \sum_{j=1}^{N} e^{i\left(\vartheta_{j}(t)-\vartheta_{H}(t)\right)} .
$$

Fix $\omega$ and $v$. Given a small $\sigma>0$ there exist large enough $N$ and $\beta$ such that for an interval of coupling $K$ there exist attracting asynchronous and synchronous states where for any $t$ large enough:

- if $\gamma(t)$ starts close to an asynchronous state for $K<K_{c}^{f}$, then $|r(\gamma(t))|<\sigma$.

- if $\gamma(t)$ starts close to a synchronous state for $K>K_{c}^{b}$, then $|r(\gamma(t))|>1-\sigma$.

Moreover, inside the interval $\left(K_{c}^{b}, K_{c}^{f}\right)$ both macroscopic states coexist.

\subsubsection{Discussions}

The result relies on a finite large number of leaves $N$ like a thermodynamic limit. Two underlying concepts to prove the result are Möbius group reduction and normal hyperbolicity. Definition and discussion of examples are found in Chapter 3 and Chapter 4 , for normal hyperbolicity and Möbius group reduction respectively. Below we introduce informally their meaning and discuss meaningfully the physical meaning behind the order parameter discontinuity.

Möbius group reduction. A class of coupled oscillators setup are described by a system of $N$ equations of motion expressed in a closed form:

$$
\dot{\phi}_{j}=f e^{i \phi_{j}}+g+\bar{f} e^{-i \phi_{j}}, \quad j=1, \ldots, N,
$$

where $f$ and $g$ are smooth complex and real-valued function, respectively. They are function of the phases and do not depend on subscript $j$. Consequently, on one hand, 
all oscillators behave identically because rotate with the same natural frequency. On the other hand, due to the global sinusoidal coupling, every oscillator feels the influence of all others. In a macroscopic view, it is a mean-field problem. Each oscillator supplies the mean-field and simultaneously are subjected to its effect.

The mean-field is a three-dimensional dynamical observable which predicts the collective behavior of the oscillators asymptotically, instead of $N$-dimensional as the original problem. So, one reduced to a low-dimensional description being now constrained to parameters namely complex $\alpha$ and phase $\psi$. They are group parameters of a Möbius transformation that preserves the unit circle:

$$
\mathcal{F}_{\alpha, \psi}\left(e^{i \theta_{j}}\right)=\frac{e^{i \psi}\left(e^{i \theta_{j}}\right)+\alpha}{1+\bar{\alpha} e^{i \psi}\left(e^{i \theta_{j}}\right)} .
$$

The ordinary differential equations on the Möbius group are described by :

$$
\begin{array}{ll}
\dot{\alpha}=i\left(f \alpha^{2}+g \alpha+\bar{f}\right) & \\
\dot{\psi}=f \alpha+g+\bar{f} \bar{\alpha} & \\
\dot{\theta}_{j}=0, & j=1, \ldots, N,
\end{array}
$$

denoting a reduced system. The missing $N-3$ dimensions are constants of motion called cross-ratios. These function are invariant by Möbius group action.

The system (1.1) under study can be written in terms of the order parameter introduced at Equation (1.2). The order parameter depends on the state of each oscillator and influences the dynamics of each one as well. Consequently, order parameter measures the mean-field dynamics. We obtain in our result that order parameter and $\alpha$ have similar time evolution when $\theta$ 's are selected appropriately. Thus, we are able to predict the collective behavior analyzing order parameter asymptotic dynamics for varying coupling strength.

Normal hyperbolicity. Let a surface on the phase space which any solution starting in such surface remains there for all time. This surface is called an invariant manifold. Normal hyperbolicity is a dynamical property that states: under the linearized dynamics, the convergence rate transverse to the invariant manifold dominates any convergence rate of orbits lying on the manifold. The phase space is decomposed only in contracting or/and expanding directions about such manifold. The remarkable consequence is: small perturbations on the vector field denoting the dynamics does not affect this outstanding decay nature, the so-called persistence against perturbations.

The synchronous state on the diffusely coupled oscillators system establishes a manifold which for appropriate conditions are normally hyperbolic, see at Pereira.(9) Our result checks that the two macroscopic states, asynchronous and synchronous states, define normally hyperbolic invariant manifolds and varying the coupling strength, their attracting condition alters. 


\subsubsection{Sketch of the proof}

The strategy involves the steps below:

First step: Synchronous manifold determination. Since the coupling depends only in the phase difference of two oscillators in system (1.1), coherent state forms a phase locking manifold. Under certain conditions, we verify that the $M_{\text {sync }}$ manifold is invariant and hyperbolic (attracting) as function of coupling strength.

Second step: Asynchronous manifold determination. The system (1.1) is $N+1$ dimensional of nonidentical oscillators, because the isolated dynamics of the hub and a leaf are different (due to the parameter $\beta$ be larger than one). In order to deal with this issue, we consider the phase difference $\phi_{k}=\vartheta_{k}-\vartheta_{H}$ system. In this referential frame, it is a $N$-dimensional of identical oscillators since hub dynamics was taken into account on each leaf. Thus, we write in the form of the Equation (1.3) and the Möbius group technique can be applied.

The case where the number of oscillators is large and the phases are roughly equally spread over the unit circle defines a open set of initial conditions which implies the $\alpha$ equation is solvable. We determine hyperbolic conditions on the invariant manifold under the flow of $\alpha$ equation (with $v \in(0, \pi / 4)$ ), which lies on $\mathbb{C} \times S^{1}$ because $\psi$ is driven by $\alpha$ in initial conditions used.

Final step: Persistence of attracting invariant manifold. We check that order parameter $r$ is $C^{k}$ close to $\alpha$ for the previous chosen initial conditions. So, we have order parameter dynamics as a small perturbation of $\alpha$ dynamics. Consequently, using normal hyperbolicity theory, we assure that taking the set of all initial conditions roughly spread over the unit circle yields the asynchronous manifold $M_{\text {async }}$ in $\mathbb{C} \times S^{1} \times \mathbb{T}^{N}$ which is normally attracting invariant manifold under the flow of order parameter equation. Moreover, there is the synchronous $M_{\text {sync }}$ achieved in the first step.

With this in hand, finally we look at $\beta$ large. It guarantees that order parameter assumes different values when solution curves are plugged in and measured for each invariant manifold. Organization. The present work is organized as follows: in Chapter 2 we provide the background theory and notation setting that will be adopted throughout this dissertation. Normal hyperbolicity is discussed in Chapter 3 and Möbius group reduction on identical oscillators in Chapter 4 . Chapter 5 is devoted to present the proof of our result and we finish with Conclusions in Chapter 6. 



\section{Lie Groups and Projective Geometry}

In this chapter we introduce definitions and notation setting adopted throughout Normal hyperpobilicity theory in Chapter 3, Möbius group reduction in Chapter 4 and main theorem proof. The concepts lie on Differential Geometry running from embedded Manifolds on $\mathbb{R}^{n}$, Lie Groups and their action on Manifolds to Möbius group geometry and Lie theorem on infinitesimal generators.

The depth of discussion in each topic is concise, thus all examples and remarks are balanced based on what reader should know before hand to understand the main theorem. If the reader desires deeper knowledge than what is shown here, appropriate literature will be indicated.

\subsection{Manifolds}

In a heuristic scenario, there are two ways to define manifold: an abstract or a concrete manner. Here we shall use the concrete way because fits the requirement for all theorems, forthcoming theory and our result. See Warner (10) for the abstract definition.

We see a manifold as subset of $\mathbb{R}^{n}$ satisfying some differentiable structure, generalizing curves and surface geometric objects. In this way we define 2.1.1:

Definition 2.1.1 ( $C^{k}$-manifold). A subset $M \subset \mathbb{R}^{n}$ of dimension $m$ is called a $C^{k}$ manifold if

- Let $\mathcal{I}$ a countable index set. There exists a countable collection of open sets $V_{l} \subset \mathbb{R}^{n}$ with $U_{l}=V_{l} \cap M$ and with $l \in \mathcal{I}$ such that $M=\bigcup_{l \in \mathcal{I}} U_{l}$.

- There exists a $C^{k}$ diffeomorphism $\Psi_{l}$ defined on each $U_{l}$ onto some open set in $\mathbb{R}^{m}$.

- For all $l, j \in \mathcal{I}: \Psi_{j} \circ \Psi_{l}^{-1}: \Psi_{l}\left(U_{l} \cap U_{j}\right) \rightarrow \Psi_{j}\left(U_{l} \cap U_{j}\right) \in C^{k}$.

The terminology smooth indicates differentiability of class $C^{\infty}, k=\infty$. In addition, the degree of differentiability of the manifold is the same as the degree of differentiability of all the $\Psi_{l}$ 's, that is the minimum of all of them.

Once we introduced the concept of a manifold, note that

- Let $M$ be a $m$-dimensional manifold and $U \subset M$, the we say $f: U \rightarrow \mathbb{R}$ is a $C^{\infty}$ function on $U$ if for each $\Psi$ on $M: f \circ \Psi^{-1}$ is $C^{\infty}$. 
- A map o between manifolds is useful to be considered. Let $M_{1}$ and $M_{2}$ be smooth manifolds, the map $o: M_{1} \rightarrow M_{2}$ is a smooth map wherever $o$ is a smooth function $C^{\infty}$ taking open subsets on $M_{1}$ to $M_{2}$.

Example 2.1.2. $\quad$ - Let $V$ be a finite dimensional real vector space. $V$ has a natural manifold structure. It includes the complex $n$-space $\mathbb{C}^{n}$, which is a real $2 n$ dimensional vector space.

- Let $F: I \rightarrow \mathbb{R}$ be a $C^{r}$ function where $I \subset \mathbb{R}$ is some open set. Then the graph of $f$ is defined as follows:

$$
\text { graph } F:=\left\{(x, y) \in \mathbb{R}^{2} \mid y=F(x), x \in I\right\}
$$

Let $U=\mathbb{R}^{2} \cap$ graph $F$ implies $U=\operatorname{graph} F$. And $\Psi: U \rightarrow I,(x, F(x)) \mapsto x$. $\Psi$ is $C^{r}$ since $F$ is $C^{r}$. Therefore, graph $F$ is a one-dimensional $C^{r}$ manifold.

- Let $S^{1} \subset \mathbb{R}^{2}$ and choose the following

$$
\begin{array}{ll}
U_{1}=S^{1} \cap\{y>0\} & \Psi_{1}(x, y)=x \\
U_{2}=S^{1} \cap\{x<0\} & \Psi_{2}(x, y)=y \\
U_{3}=S^{1} \cap\{y<0\} & \Psi_{3}(x, y)=x \\
U_{4}=S^{1} \cap\{x>0\} & \Psi_{4}(x, y)=y
\end{array}
$$

where $\Psi_{1} \neq \Psi_{3}$ and $\Psi_{2} \neq \Psi_{4}$, since they are defined on different domains. For instance we look at a composition map

$$
\begin{aligned}
U_{1} \cap U_{2} & =S^{1} \cap\{y>0 \cap x<0\} \\
\left(\Psi_{2} \circ \Psi_{1}^{-1}\right)(x) & =\Psi_{2}\left(x, \sqrt{1-x^{2}}\right) \\
& =\sqrt{1-x^{2}} \in C^{\infty} \text { on } \Psi_{1}\left(U_{1} \cap U_{2}\right)=(-1,0) .
\end{aligned}
$$

We introduce the concepts of Tangent spaces based on curves on a manifold, which lead us to the study of differential equations. Let a $m$-dimensional manifold $M, U \subset M$ and $\Psi: U \rightarrow \mathbb{R}^{m}$. Consider a curve $\gamma:(-\delta, \delta) \subset \mathbb{R} \rightarrow M, \gamma \in C^{1}$ where we verify that $\Psi \circ \gamma:(-\delta, \delta) \rightarrow \mathbb{R}^{m}$ and a point $p=\gamma(0) \in U$. The vector tangent to $\gamma$ at $p$ is

$$
\mathbf{v}=\left.\frac{d}{d t}(\Psi \circ \gamma)(t)\right|_{t=0} \in \mathbb{R}^{m}
$$

where $v$ is associated to the pair $(U, \Psi)$ chosen. We are able to set a linear homeomorphism between another possible choice. Then $v$ is a coordinate representation of an abstract tangent vector $\gamma^{\prime}(0)$ at point $p$. 
Definition 2.1.3. Let a $m$-dimensional manifold $M$ and $\delta>0$. Consider a curve $\gamma$ : $(-\delta, \delta) \subset \mathbb{R} \rightarrow M, \gamma \in C^{1}$ where a point $p=\gamma(0)$. Then the tangent space at point $p$ is the set

$$
T_{p} M=\{p\} \times\{\gamma /(0)\}
$$

with $\gamma \prime(0)$ raging over all possible values, as we vary over all such curves $\gamma$. Each element of $T_{p} M$ is called a tangent vector at $p$ and denoted $X_{p}$.

Example 2.1.4. $\quad$ Consider trivially $M=\mathbb{R}^{m}$. The set $T_{x} \mathbb{R}^{m}$ is identified with $\mathbb{R}^{m}$ itself. Every element $v \in \mathbb{R}^{m}$ can be understood as a tangent vector of a $C^{1}$ curve, after moving its initial point $0 \in \mathbb{R}^{m}$ to $p \in \mathbb{R}^{m}$. Take the curve $\gamma(t)=p+t$, $\mathrm{v} \in \mathbb{R}^{m}$ then $d \gamma(t) /\left.d t\right|_{t=0}=\mathrm{v}$.

- Consider $F: \mathbb{R}^{m} \rightarrow \mathbb{R}^{n-m}, M=\operatorname{graph} F=\left\{(x, y) \in \mathbb{R}^{n} \mid y=F(x)\right\}$ and $\delta>0$. As we have seen $U=M$ and $\Psi: U \rightarrow \mathbb{R}^{m}$ with $(x, y) \in U \mapsto x \in \mathbb{R}^{m}$. Let $g:(-\delta, \delta) \rightarrow \mathbb{R}^{m}, g(0)=x$ and $\Psi^{-1}(x)=(x, F(x))=p$ so

$$
\begin{aligned}
T_{p} M & =\{(x, F(x))\} \times\left\{D \Psi^{-1}(x) \cdot g \prime(0) \mid g \in C^{1}\left((-\delta, \delta), \mathbb{R}^{m}\right)\right\} \\
& =\{(x, F(x))\} \times\left\{(\mathrm{v}, D F(x) \mathrm{v}) \mid \mathrm{v} \in \mathbb{R}^{m}\right\}
\end{aligned}
$$

There is a normal space to the manifold at point $p$ as well. We define it

Definition 2.1.5 (Normal space at a point). For a point $p \in M \subset \mathbb{R}^{n}$ the normal space is the set

$$
N_{p}=\left\{v \in \mathbb{R}^{n} \mid v \perp T_{p} M\right\}
$$

wherever be orthogonal is defined based on the standard inner product for $\mathbb{R}^{n}$. The direct sum of both spaces at point $p$ yields the whole space

$$
T_{p} \mathbb{R}^{n}=T_{p} M \oplus N_{p}
$$

A natural generalization of tangent space and normal space at a point is extend it to different points of the manifold, named tangent bundle $T M$ and normal bundle $N$. However we do not need dive into them.

Remark 2.1.6. The smooth map $\Psi$ with open set $U \subset M$ forms what is called a coordinate system $(U, \Psi)$ of a $m$-dimensional smooth manifold. From here on we think of the map $\Psi$ as specifying local coordinate functions $x_{1}, \ldots, x_{m}$ where for each $j \in\{1, \ldots, m\}$ and $p \in M: x_{j} \in C^{\infty}(U, \mathbb{R})$ with $x_{j}(p)=\Psi(p)_{j}$ (the $j$-th component of $\Psi$ ).

Definition 2.1.7. A vector field $X$ is a map that assigns to each point $p \in M$ a tangent vector $X_{p} \in T_{p} M$. 
Choosing a local coordinate system $x_{1}, \ldots, x_{m}$ and smooth functions $f_{j} \in C^{\infty}(M, \mathbb{R})$, a smooth vector field can be expressed as

$$
X_{p}=\sum_{j=1}^{m} f_{j}(p) \frac{\partial}{\partial x_{j}}
$$

Let a vector field $X$ and a curve $\gamma \in C^{\infty}((-\delta, \delta) \subset \mathbb{R}, M)$, then $\gamma$ is called an integral curve (or solution curve) for $X$ if for each $t \in(-\delta, \delta)$ we have

$$
\frac{d}{d t} \gamma(t)=X_{\gamma(t)}
$$

The case the manifold is $\mathbb{R}^{m}$ there is ordinary differential equations system as below:

$$
\frac{d x_{k}}{d t}=f_{k}\left(x_{1}(t), \ldots, x_{m}(t)\right)
$$

where results such as existence and uniqueness theorem is valid, see for example Chicone (11) or Teschl.(12) Whether the integral curves exist for all $t \in \mathbb{R}$ and for all $p \in M$ we say the vector field is complete and defines a flow.

Definition 2.1.8 (Flow). Consider a $m$-dimensional manifold $M$ and an integral curve $\gamma$ of the vector field $X$ passing through $p \in M$. There exists the map called flow

$$
\begin{gathered}
\varphi: \mathbb{R} \times M \rightarrow M \\
(t, p) \mapsto \varphi(t, p),
\end{gathered}
$$

generated by $X$. We shall denote $\varphi(t, p)=\varphi^{t}(p)$. The properties are

$$
\begin{array}{ll}
p \in M: & \varphi^{0}(p)=p \\
p \in M: & \varphi^{t}\left(\varphi^{s}(p)\right)=\varphi^{t+s}(p), \quad \forall t, s \in \mathbb{R} .
\end{array}
$$

Moreover, if $p=\gamma(0)$ then $\varphi^{t}(p)=\gamma(t)$, so $\forall t \in \mathbb{R}$ where the integral curve $\gamma$ is defined: $\frac{d}{d t} \varphi^{t}(p)=X_{\varphi^{t}(p)}$.

From here on we will denote a point on a manifold $M$ as $x \in M$ instead of $p$ since usually $M$ will be $\mathbb{R}^{n}$ or $\mathbb{C}^{n}$ for some $n$. 


\subsection{Lie Groups and Möbius transformation}

In this section, we will introduce Lie groups using what we explored in previous section 2.1. We should first introduce the definition of a Group:

Definition 2.2.1 (Groups). A nonempty set of elements $G$ is said to form a group if in $G$ is defined a binary operation, called the product and denoted by $\cdot$, such that the following hold

i. Closed: $g_{1}, g_{2} \in G$ implies that $g_{1} \cdot g_{2} \in G$.

ii. Associative law: $g_{1}, g_{2}, g_{3} \in G$ implies that $g_{1} \cdot\left(g_{2} \cdot g_{3}\right)=\left(g_{1} \cdot g_{2}\right) \cdot g_{3}$.

iii. Existence of an identity element: there exists $\mathbf{1}_{G} \in G$ such that $\forall g_{1} \in G: g_{1} \cdot \mathbf{1}_{G}=$ $\mathbf{1}_{G} \cdot g_{1}=g_{1}$.

iv. Existence of a inverse element: for every $g_{1} \in G$ there exists an element $g_{1}^{-1} \in G$ such that $g_{1} \cdot g_{1}^{-1}=g_{1}^{-1} \cdot g_{1}=\mathbf{1}_{G}$.

If $H$ a nonempty subset of $G$ is a group under the operation defined in $G$, then $H$ is called a subgroup of $G$. We denote $H \subset G$. Focusing our discussion on Lie groups we introduce them as well:

Definition 2.2.2. An $m$-parameter Lie group is a group $G$ which also carries the structure of an $m$-dimensional smooth manifold in such a way that both the group operation

$$
o: G \times G \rightarrow G, g_{1}, g_{2} \in G: \quad o\left(g_{1}, g_{2}\right)=g_{1} \cdot g_{2}
$$

and the inversion

$$
i: G \rightarrow G, g \in G: \quad i(g)=g^{-1}
$$

are smooth maps between manifolds.

Example 2.2.3. - The set of all matrices $\mathbb{R}^{n \times n}$ (or $\mathbb{C}^{n \times n}$ ) with determinant different from zero under the usual matrix multiplication forms a group called $G L(n, \mathbb{R})$ (or $G L(n, \mathbb{C})$ ). The inverse exists since the determinant is different from zero. In particular for $n=2$, we denote the general linear group as $G L(2, \mathbb{R})$ (or $G L(2, \mathbb{C})$ ). If we identify the points of $\mathbb{R}^{n^{2}}\left(\mathbb{R}^{4 n^{2}}\right)$ with the $\mathbb{R}^{n \times n}\left(\mathbb{C}^{n \times n}\right)$ we have also that $G L(n, \mathbb{R})(G L(n, \mathbb{C}))$ is a $n^{2}$-dimensional $\left(4 n^{2}\right.$-dimensional) manifold.

- Consider a particular case from the previous examples, where the determinant is equal to 1 . We have the special linear group. For the case $n=2$ we denote $S L(2, \mathbb{C})=\left\{A \in \mathbb{C}^{2 \times 2} \mid \operatorname{det} A=1\right\}$. Thus it holds $S L(2, \mathbb{C}) \subset G L(2, \mathbb{C})$. 
Definition 2.2.4 (Group action). Let $G$ be a group and $M$ a manifold. The (left) group action of $G$ on $M$ is a function

$$
\begin{aligned}
\varrho: G \times M & \rightarrow M \\
(g, x) & \mapsto \varrho(g, x) .
\end{aligned}
$$

We denote $\varrho(g, x)$ as $g \cdot x$. For all $g, h \in G$ and all $x \in X$ the properties :

$$
e \cdot x=x \quad(g h) \cdot x=g \cdot(h \cdot x) \quad g \cdot\left(g^{-1} \cdot x\right)=x
$$

must be satisfied.

For each $g \in G$, the function $\varrho^{g}: M \rightarrow M$ maps $x$ to $\varrho^{g}(x)=g \cdot x$ and is bijective where the inverse map is $\left(\varrho^{g}\right)^{-1}: x \mapsto g^{-1} \cdot x$. For all cases we assume that the map is a diffeomorphism.

Example 2.2.5. - The flow introduced at Definition 2.1.8 has the same properties of a group action on a manifold. For this reason often it is called one-parameter group of transformation.

- Let $\left\{e_{i}: i=1, \ldots, n\right\}$ be the canonical basis of $\mathbb{C}^{n}$, where $e_{i}$ is the $n$-tuple consisting of all zeros except for a 1 in the $i$ th component. Each matrix $A \in G L(n, \mathbb{C})$ uniquely determines a linear transformation on $\mathbb{C}^{n}$

$$
A\left(e_{j}\right)=\sum_{i} A_{i j} e_{i}
$$

where $\left(A_{i j}\right)$ represents the matrix element of $A$ with respect to the basis $e_{i}$. $A$ acts by matrix multiplication when we consider the complex $n$-tuples of $\mathbb{C}^{n}$ as $n \times 1$ matrices.

\subsubsection{Möbius group}

Also we consider general linear group acting on the projective complex space. The projective space of a vector space is defined as the set of all lines through the origin. Namely, we denote by $\mathbb{C P}^{1}=\mathbb{P}\left(\mathbb{C}^{2}\right)$ the projective complex space.

Such space is the set of points $0 \neq(u, v) \in \mathbb{C}^{2}$ where they share an equivalence relation: $\left[\begin{array}{l}u_{1} \\ v_{1}\end{array}\right]=\left[\begin{array}{l}a u \\ a v\end{array}\right]$ when there exists $a \in \mathbb{C} \backslash\{0\}$. We define the homogeneous coordinate $\left[\begin{array}{l}u \\ v\end{array}\right] \in \mathbb{C P}^{1}$ of the line passing through the origin and $0 \neq(u, v) \in \mathbb{C}^{2}$. The projective coordinate $z=u / v$ is obtained when we write $a=1 / v$ with $v \in \mathbb{C} \backslash\{0\}$ and the homogeneous coordinate is $\left[\begin{array}{l}z \\ 1\end{array}\right]$. If we include $v=0$, the projective complex line identifies with the extended complex plane $\mathbb{C}, z \in \mathbb{C} \cup\{\infty\}=: \hat{\mathbb{C}}=\mathbb{C P}^{1}$. 
The action $G L(2, \mathbb{C}) \times \mathbb{C P}^{1} \rightarrow \mathbb{C P}^{1}$ is given by matrix multiplication with complex coefficients $a, b, c, d \in \mathbb{C}$ and $a d-b c \neq 0$ :

$$
\left[\begin{array}{l}
u^{\prime} \\
v^{\prime}
\end{array}\right]=\left(\begin{array}{ll}
a & b \\
c & d
\end{array}\right)\left[\begin{array}{l}
u \\
v
\end{array}\right] .
$$

The result in projective coordinate

$$
z^{\prime}=u^{\prime} / v^{\prime}=\frac{(a u+b v)}{(c u+d u)}=\frac{(a z+b)}{(c z+d)}
$$

Thus, for a fixed group element $A$ it is a diffeomorphism (actually analytic) mapping between $\hat{\mathbb{C}}$ to itself forming a linear fractional transformation called Möbius transformation: $\mathcal{F}_{a b c d}: \hat{\mathbb{C}} \rightarrow \hat{\mathbb{C}}$ such that $\mathcal{F}_{a b c d}(z):=\frac{a z+b}{c z+d}$, where $a, b, c, d \in \mathbb{C}$ and $a d-b c \neq 0$. Besides let the composition of functions $\mathcal{F}_{a_{1} b_{1} c_{1} d_{1}} \circ \mathcal{G}_{a_{2} b_{2} c_{2} d_{2}}(z)=\mathcal{F}_{a_{1} b_{1} c_{1} d_{1}}\left(\mathcal{G}_{a_{2} b_{2} c_{2} d_{2}}(z)\right)$. The set of all linear fractional transformations forms a group under the operation of composition of functions. We denote as $\mathcal{M}:=\left\{\mathcal{F}_{a b c d} \mid a d-b c \neq 0\right\}$.

Note that multiples of the identity matrix $a \mathbf{1}$ of $G L(2, \mathbb{C})$ act on $\mathbb{C P}^{1}$ as the identity, since $a$ cancels out in the fractional form. Thus, we introduce $P G L(2, \mathbb{C})=G L(2, \mathbb{C}) /\{a \mathbf{1}\}$ named projective general linear group, leading to $P G L(2, \mathbb{C}) \cong \mathcal{M}$. See at Herstein (13) or Olver (14) for details.

Consider $n \in \mathbb{N}$ denoting the dimension of $\hat{\mathbb{C}}^{n}$ and the projective complex space with $\left[\begin{array}{l}u \\ v\end{array}\right]=\left[\left[\begin{array}{l}u_{1} \\ v_{1}\end{array}\right], \ldots,\left[\begin{array}{l}u_{n} \\ v_{n}\end{array}\right]\right] \in\left(\mathbb{C P}^{1}\right)^{n}$ or $z=\left(z_{1}, \ldots, z_{n}\right) \in \hat{\mathbb{C}}^{n} . \mathcal{M}$ acts diagonally on these spaces as following

$$
\mathcal{F}_{a b c d}(z)=\left(\mathcal{F}_{a b c d}\left(z_{1}\right), \ldots, \mathcal{F}_{a b c d}\left(z_{n}\right)\right)
$$

the same in homogeneous coordinates. Let $n \geq 3$ and $z, w$ be $N$-tuples with all coordinates distinct. There exists a unique element $\mathcal{F}_{a, b, c, d}$ such that for $i \in\{1, \ldots, N\}$ : $w_{i}=\mathcal{F}_{a, b, c, d}\left(z_{i}\right)$. Furthermore, Möbius group preserves cross-ratios.

Definition 2.2.6. Let $z_{1}, z_{2}, z_{3}, z_{4} \in \hat{\mathbb{C}}$ be four distinct numbers and define the function $C_{1,2,3,4}: \hat{\mathbb{C}}^{4} \rightarrow \mathbb{C}$ such that

$$
C_{1,2,3,4}(z)=\frac{\left(z_{1}-z_{3}\right)}{\left(z_{1}-z_{4}\right)} \frac{\left(z_{2}-z_{4}\right)}{\left(z_{2}-z_{3}\right)}
$$

Let $w_{i}=\mathcal{F}_{a b c d}\left(z_{i}\right)$ for $i=1, \ldots, 4$. Then we have 


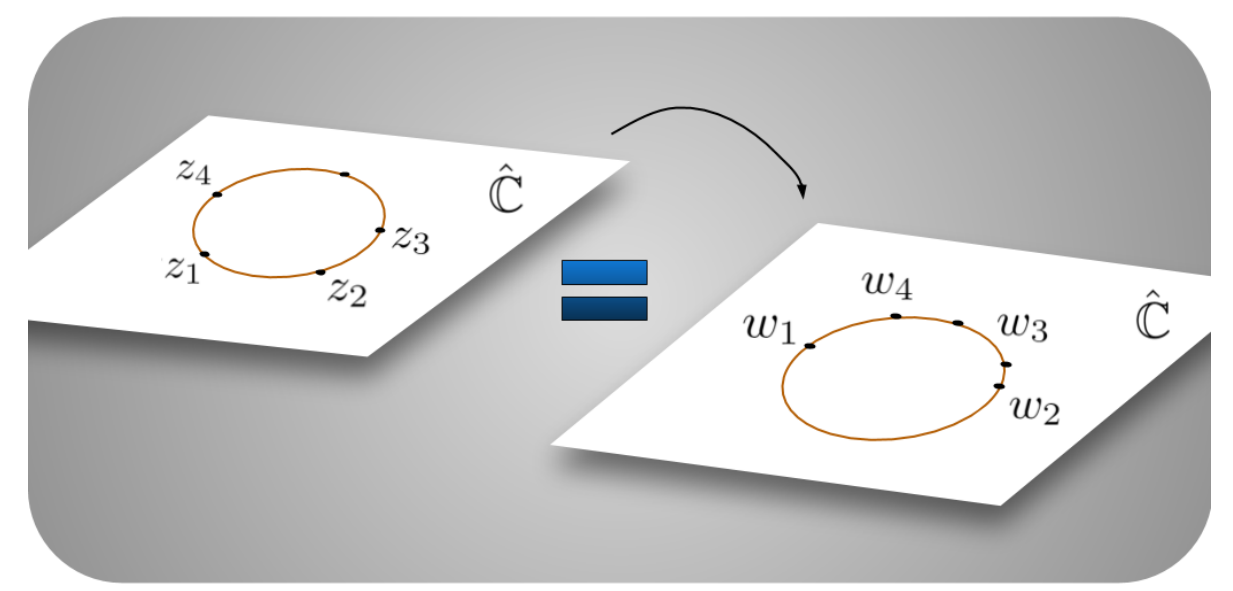

Figure 2 - Four-distinct points define unique circle passing through them and a Möbius transformation which maps $z$ 's to $w$ 's. Cross-ratio of both set of points is equal.

Source: By the author.

$$
C_{1,2,3,4}(w)=C_{1,2,3,4}(z)
$$

This can be explained as follows: let $\left(z_{2}, z_{3}, z_{4}\right)$ be a triple with distinct coordinates. There exists a unique Möbius transformation that maps $\left(z_{2}, z_{3}, z_{4}\right)$ to $(1,0, \infty)$ given by

$$
\mathcal{H}(z)=\frac{\left(z-z_{3}\right)}{\left(z-z_{4}\right)} \frac{\left(z_{2}-z_{4}\right)}{\left(z_{2}-z_{3}\right)}
$$

The right hand side is the cross-ratio of $C_{1,2,3,4}(z)$. On the other hand, the four distinct points $(\mathcal{H}(z), 1,0, \infty)$ define a cross-ratio as well:

$$
C_{\mathcal{H}(z), 1,0, \infty}(z)=\frac{(\mathcal{H}(z)-0)}{(\mathcal{H}(z)-\infty)} \frac{(1-\infty)}{(1-0)}=\mathcal{H}(z)
$$

Thus, both cross-ratios are equal. We can construct a Möbius transformation $\mathcal{G}$ from $(1,0, \infty)$ to a triple with distinct coordinates $\left(w_{2}, w_{3}, w_{4}\right)$. The above argument about cross-ratios is similar to the inverse of $\mathcal{G}$. Thus, the composition between $\mathcal{G}$ and $\mathcal{H}$ is a Möbius transformation $\mathcal{F}_{a b c d}$ with $w_{i}=\mathcal{F}_{a b c d}\left(z_{i}\right)$ for $i=1, \ldots, 4$, which preserves the cross-ratio. Figure 2 displays Möbius group action on four-distinct points which lie on the same circle.

Cross-ratio can also be defined to $n \in \mathbb{N}$ with $n \geq 4$. Let $z=\left(z_{1}, \ldots, z_{n}\right)$ be a $n$-tuple and select four-distinct coordinates of it. We denote for $p, q, r, s \in\{1, \ldots, n\}$ : 


$$
\begin{aligned}
& C_{p, q, r, s}: \hat{\mathbb{C}^{n}} \rightarrow \mathbb{C} \\
& C_{p, q, r, s}(z)=\frac{\left(z_{p}-z_{r}\right)}{\left(z_{p}-z_{s}\right)} \frac{\left(z_{q}-z_{s}\right)}{\left(z_{q}-z_{r}\right)} .
\end{aligned}
$$

and given by the same Equation (2.5). In addition, only $n-3$ are functionally independent. The proof relies upon 4! cross-ratios of corresponding 4 ! permutations of $z_{p}, z_{q}, z_{r}, z_{s}$ are written as function of $C_{p q r s}$. Look at Appendix in Marvel, Mirollo and Strogatz (15) for detailed proof.

These linear fractional transformations can be decomposed in simpler transformations that have a clearer geometrical description:

1) $\mathcal{F}_{1(d / c) 01}=z+d / c$ : translation;

2) $\mathcal{F}_{0110}=1 / z$ : inversion and reflection with respect to the real axis;

3) $\mathcal{F}_{e 001}=e z$, with $e=\frac{b c-a d}{c^{2}}$ : rotation and scaling;

4) $\mathcal{F}_{1(a / c) 01}=z+a / c$ : translation.

In this way, a general Möbius transformation can be written as

$$
\mathcal{F}_{a b c d}(z)=\frac{a}{c}+\frac{b c-a d}{c^{2}}\left[\frac{1}{z+\frac{d}{c}}\right]=\frac{a z+b}{c z+d} .
$$

In the scenario, where $z \in \mathbb{C}$ and $\phi \in S^{1}: z=e^{i \phi}$, Möbius group action must preserve the unit disc since for any phase, the modulus of $z$ is always one. This implies that only Möbius group elements are allowed, that are automorphisms of the unit disc $\mathbb{D}:=\{z \in \mathbb{C}|| z \mid<1\}$. Note that this is true because disc automorphisms automatically preserve the boundary of the disc, the unit circle, which is what we are interested in. We denote by AutD the set of linear fractional transformations that maps the unit disc to itself, consequently Aut $\mathbb{D} \subset \mathcal{M}$ and Aut $\mathbb{D} \cong P S L(2, \mathbb{R})$. The elements $\mathcal{F}_{\alpha, \psi}$ are identified by two parameters, $\alpha$ and $\psi$ :

$$
\mathcal{F}_{\alpha, \psi}(z)=\frac{e^{i \psi} z+\alpha}{1+\bar{\alpha} e^{i \psi} z}
$$

for $\alpha \in \mathbb{D}$ and $\psi \in S^{1}$. To construct the transformation given by Equation (2.6), one builds its inverse transformation $\mathcal{F}_{\alpha, \psi}^{-1}$ that takes any point inside $\mathbb{D}$, namely $\alpha$, and send to the origin. After composes it to a rotation of angle $\psi$ which fixes the origin. See Stein and Shakarchi.(16) 


\subsection{Lie Theorem}

The flow generated by a vector field satisfies a differential equation in 2.1.8 and also is a group of transformation. Let a small parameter $\varepsilon \in \mathbb{R}$. $\varphi^{\varepsilon}$ is at least twice differentiable then in local coordinates

$$
\varphi^{\varepsilon}(x)=x+\varepsilon f(x)+\mathcal{O}\left(\varepsilon^{2}\right)
$$

where $f=\left(f_{1}, \ldots, f_{m}\right)$ are the coefficients of $X$ (similar to Equation (2.2)) and $X$ is called the infinitesimal generator of the group action. Conversely, the infinitesimal generator of a one parameter group of transformation $\varphi^{\varepsilon}(x)$ for $x \in M$ is

$$
X_{x}=\left.\frac{d}{d \varepsilon} \varphi^{\varepsilon}(x)\right|_{\varepsilon=0}
$$

determining an one-to-one correspondence between them. The above differential equation (2.7) suggests that the solution is given by

$$
\varphi^{\varepsilon}(x)=e^{X \varepsilon} x
$$

which all properties of a group action 2.2.4 hold when exponential function properties are taken into account. Therefore, group of transformations on a manifold are generated by infinitesimal generator, establishing vector fields to a ordinary differential equation. Such identification is the cornerstone of the Lie group theory, and named after Sophus Lie. The set of all vector fields generated by a Lie group or a group of transformations on a manifold is called a Lie algebra if it satisfies some properties. Before we get into it, let us make some considerations.

Example 2.3.1. $\quad$ - Let $M=\mathbb{R}^{m}$ with coordinate $x$ and consider the vector field

$$
X_{A}=\sum_{i=1}^{m}\left(\sum_{j=1}^{m} a_{i j} x_{j}\right) \frac{\partial}{\partial x_{i}},
$$

where $A=\left(a_{i j}\right) \in \mathbb{R}^{m \times m}$ does not depend on $x$. Then the differential equation is $\frac{d}{d \varepsilon} \varphi^{\varepsilon}(x)=A \varphi^{\varepsilon}(x)$. The flow is

$$
\varphi^{\varepsilon}(x)=e^{A \varepsilon} x
$$

where $e^{A \varepsilon}=\sum_{n=0}^{\infty} \frac{(A \varepsilon)^{n}}{n !}$ is the usual matrix exponential and $A$ is the infinitesimal generator. 
Note that $e^{A \varepsilon} \in G L(m, \mathbb{R})$ because the determinant is always different than zero, then denote the set of matrices which are infinitesimal generators

$$
\mathfrak{g l}(m, \mathbb{R}):=\left\{A \in \mathbb{R}^{m \times m} \mid e^{A \varepsilon} \in G L(m, \mathbb{R})\right\},
$$

which we define for the complex field as well, so we denote $\mathrm{F}=\mathbb{R}, \mathbb{C}$.

Before we state $\mathfrak{g l}(m, \mathrm{~F})$ Lie algebra definition notice that if $H_{J}=\{\exp J \varepsilon\} \subset$ $G L(m, \mathrm{~F})$ is a matrix one-parameter subgroup generated by $J \in \mathfrak{g l}(m, \mathrm{~F})$ and $A \in$ $G L(m, \mathrm{~F})$, then the following holds: $A^{-1} e^{J \varepsilon} A=\exp \left(A^{-1} J A \varepsilon\right)$ which yields $H_{A^{-1} J A}$ generated by the matrix $A^{-1} J A$. And more, let $A=e^{K \varepsilon} \in G L(m, \mathrm{~F})$ itself be generated by some $K \in \mathfrak{g l}(m, \mathbf{F})$. Therefore, $e^{-K \varepsilon} J e^{K \varepsilon} \in \mathfrak{g l}(m, \mathbf{F}), \forall \varepsilon$. After expanding both matrix exponential we conclude that the commutator $[J, K]=J K-K J \in \mathfrak{g l}(m, \mathrm{~F})$ and we are able to introduce Definition 2.3.2:

Definition 2.3.2. A matrix Lie algebra is a subspace $\mathfrak{g} \subset \mathfrak{g l}(m, \mathrm{~F})$ with the property that $J, K \in \mathfrak{g}$ implies $[J, K] \in \mathfrak{g}$. In particular, $\mathfrak{g l}(m, \mathrm{~F})$ is itself a matrix Lie algebra.

Remark 2.3.3. Lie algebra are abstract objects and can be defined respectively, but it carries us beyond the scope of this text. We will only use a matrix Lie algebra. See Warner (10) and Hall. (17)

Example 2.3.4. Recall the subgroup $S L(m, \mathrm{~F})$ where its associated Lie algebra is the set $\mathfrak{s l}(m, \mathbf{F})=\{J \in \mathfrak{g l}(m, \mathbf{F}) \mid \operatorname{tr} J=0\}$. Since Lie algebra forms a vector space, there is a basis. Observe that for $m=2$ a basis of $\mathfrak{s l}(2, F)$ is

$$
J_{1}=\left(\begin{array}{cc}
1 & 0 \\
0 & -1
\end{array}\right), \quad J_{2}=\left(\begin{array}{ll}
0 & 1 \\
0 & 0
\end{array}\right), \quad J_{3}=\left(\begin{array}{ll}
0 & 0 \\
1 & 0
\end{array}\right)
$$

and $S L(2, \mathrm{~F})$ generated is

$$
e^{J_{1} \varepsilon}=\left(\begin{array}{cc}
e^{\varepsilon} & 0 \\
0 & e^{-\varepsilon}
\end{array}\right), \quad e^{J_{2} \varepsilon}=\left(\begin{array}{cc}
1 & \varepsilon \\
0 & 1
\end{array}\right), \quad e^{J_{3} \varepsilon}=\left(\begin{array}{cc}
1 & 0 \\
\varepsilon & 1
\end{array}\right)
$$

Here we extend the connection between Lie group and Lie algebra, emphasizing to the case Lie group acts on a manifold. These Lie groups are generated by infinitesimal generators of the corresponding Lie algebra, and we will analyze vector fields from their action. The notation employed was chosen in order to not be confusing.

Definition 2.3.5. Let $G \subset G L(m, \mathrm{~F})$ be a matrix Lie group, acting as a transformation group on a manifold $M$. Let $J \in \mathfrak{g}$ be the infinitesimal generator of the one-parameter 
subgroup $\{\exp \varepsilon J\} \subset G$. So, we define the infinitesimal generator of the action of $G$ on $M$ as the vector field at a point $x \in M$

$$
X_{x}^{J}=\left.\frac{d}{d \varepsilon} e^{\varepsilon J}(x)\right|_{\varepsilon=0}
$$

Example 2.3.6. Consider for instance the action of $S L(2, \mathbb{R})$ on the projective real line $\mathbb{R P}^{1}$, and similarly to $G L(2, \mathbb{R})$, multiples of identity act as the identity. Thus, we introduce $P S L(2, \mathbb{R})$ projective special linear group.

$$
\begin{aligned}
& X_{x}^{J_{1}}=\left.\frac{d}{d \varepsilon} e^{J_{1} \varepsilon}\left[\begin{array}{l}
x \\
1
\end{array}\right]\right|_{\varepsilon=0}=\left.\frac{d}{d \varepsilon}\left[\frac{e^{\varepsilon} x}{e^{-\varepsilon}}\right]\right|_{\varepsilon=0}=2 x \\
& X_{x}^{J_{2}}=\left.\frac{d}{d \varepsilon} e^{J_{2} \varepsilon}\left[\begin{array}{c}
x \\
1
\end{array}\right]\right|_{\varepsilon=0}=\left.\frac{d}{d \varepsilon}[x+\varepsilon]\right|_{\varepsilon=0}=1 \\
& X_{x}^{J_{3}}=\left.\frac{d}{d \varepsilon} e^{J_{3} \varepsilon}\left[\begin{array}{l}
x \\
1
\end{array}\right]\right|_{\varepsilon=0}=\left.\frac{d}{d \varepsilon}\left[\frac{x}{1+\varepsilon x}\right]\right|_{\varepsilon=0}=-x^{2} .
\end{aligned}
$$

Finally we see that vector fields generated by the transformation group action on the manifold can be written as

$$
X_{x}^{J}=\sum_{i=1}^{m} f_{J}^{i}(x) \frac{\partial}{\partial x^{i}}, \text { where } f_{J}(x)=\left.\frac{d}{d \varepsilon} e^{\varepsilon J} x\right|_{\varepsilon=0} .
$$

The coefficients functions $f_{J}(x)=\left(f_{J}^{1}(x), \ldots, f_{J}^{m}(x)\right)$ are coordinates to the vector field on the manifold. The associated one-parameter group of transformation $e^{J \varepsilon}(x)$ is the flow generated by $f_{J}$ vector field. The orbit on the manifold is

Definition 2.3.7. An orbit of a given point $x \in M$ is the set $\{g \cdot x \mid g \in G\}$,

which for our case is denoted by $G \cdot x=\left\{e^{J \varepsilon} x \mid \varepsilon \in \mathbb{R}\right\}$ through $x \in M$. The introduced new concepts are intrinsically identical to the theory of autonomous first order ordinary differential equations. So from uniqueness theorem for ODE, we realize that the one-parameter group of transformation generated has one-to-one correspondence to the vector field. With this in hand, Theorem 2.3.8 due to Lie can be stated

Theorem 2.3.8. Let $G$ act on $M$ and let $x \in M$. The vector space $\left.V\right|_{x}=\left\langle X_{x}^{J}\right\rangle=$ $\operatorname{span}\left\{f_{J}(x) \mid J \in \mathfrak{g}\right\}$ spanned by all the vector fields determined by the infinitesimal generators at $x$ coincides with the tangent space to the orbit $G \cdot x$ of $G$ that passes through $x$. In particular, the dimension of $G \cdot x$ equals the dimension of $\left.V\right|_{x}$. 
Example 2.3.9. Consider the vector field spanned by

$$
\begin{gathered}
f^{1}=1, \quad f^{2}=2 x, \quad f^{3}=-x^{2} \\
\dot{x}=-x^{2}+2 x+1 .
\end{gathered}
$$

Examining Equation (2.14) it follows from Theorem 2.3.8 that orbits are generated by $\operatorname{PSL}(2, \mathbb{R})$ acting on $\mathbb{R} P^{1}$ and have the form

$$
P S L(2, \mathbb{R}) \cdot x=\left\{\frac{a x+b}{c x+d} \mid a, b, c, d \in \mathbb{R}: a d-b c=1\right\} .
$$

when projective coordinates are chosen on $\mathbb{R} \mathbb{P}^{1}$.

To extend to a system of ordinary differential equations with $x \in \mathbb{R}^{n}$ and $f^{i} \in$ $C^{1}\left(\mathbb{R} \times \mathbb{R}^{n}, \mathbb{R}\right)$

$$
\dot{x}^{i}=f^{i}(t, x), \quad i=1, \ldots, n,
$$

we must define the concept of fundamental system of solutions.

Definition 2.3.10 (Fundamental system of solutions). The system of equations (2.18) has a fundamental system of solutions if the solution of (2.18) can be written in terms of a finite number $m \in \mathbb{N}$ of arbitrarily chosen particular solutions curves

$$
x_{k}(t)=\left(x_{k}^{1}(t), \ldots, x_{k}^{n}(t)\right), \quad k=1, \ldots, m .
$$

by expressions of the form

$$
x^{i}=F^{i}\left(x_{1}, \ldots, x_{m}, C_{1}, \ldots, C_{n}\right),
$$

which contain $n$ arbitrary constants $C_{1}, \ldots, C_{n}$.

Theorem 2.3.11 states a nonlinear superposition method to find general solutions for a system of differential equations, see Ibragimov.(18)

Theorem 2.3.11 (Fundamental Theorem of Vessiot-Guldberg-Lie). The system of equations (2.18) has a fundamental system of solutions if and only if it can be represented in the form

$$
\dot{x}_{i}=c_{1}(t) f_{1}^{i}(x)+\cdots+c_{r}(t) f_{r}^{i}(x)
$$


such that the operators

$$
X_{\alpha}=f_{\alpha}^{i} \frac{\partial}{\partial x^{i}} \quad \alpha=1, \ldots, r,
$$

form an $r$-dimensional Lie algebra. Moreover the number $m$ of the necessary particular (fundamental) solutions satisfies the condition

$$
n m \geq r
$$

A example of an application of Theorem 2.3.11 given by Lie is the Riccati equation with $P, Q, R: \mathbb{R} \rightarrow \mathbb{R}$ and $x \in \mathbb{R}:$

$$
\dot{x}=P(t)+Q(t) x+R(t) x^{2},
$$

spanned by the vector field indicated at Equation (2.16). It has the form of Equation (2.21), so the inequality (2.23) holds $m \geq 3$, since the Lie algebra $\mathfrak{s l}(2, \mathbb{R})$ is threedimensional $(r=3)$.

From Theorem 2.3.8, Möbius group action on the projective space is generated by Riccati equation. Consequently, the solution is written in the fundamental solution form $(2.20)$ :

$$
y(t)=\frac{a(t) x+b(t)}{c(t) x+d(t)}
$$

where $a, b, c, d$ are the group parameters of $\operatorname{PSL}(2, \mathbb{R}) \cong$ AutD obeying particular solutions of Equation (2.24) - for instance, see at Stewart (19) how their differential equations look like - and $x$ is constant in time. Besides, cross-ratio is constant along the orbit in the projective space determining an equation with four solutions of Equation (2.24). So, the equality holds $m=3$.

In the particular case $n \geq 3$, Möbius group acts diagonally on projective space and establishes closed differential equations for the parameters. In this way, one reduces the system to lower dimension (from $n$ to three). This fact will be used at Chapter 4 . 


\section{Normal hyperbolicity}

In this chapter normal hyperbolicity theory is discussed more precisely. We characterize invariant manifolds and exponential stability. Afterwards, we recapitulate some properties derived from linear autonomous system and apply these to analyze hyperbolic fixed points in nonlinear systems. This builds up the setting to state Theorem 3.0.11 on persistence of normally hyperbolic invariant manifolds. Last section is devoted to describe macroscopic states, namely synchronous and asynchronous states, in terms of the order parameter.

Invariant Manifold. Let $x \in \mathbb{R}^{n}$ and $f \in C^{1}\left(\mathbb{R}^{n}, \mathbb{R}^{n}\right)$. An autonomous system is described by

$$
\dot{x}=f(x) .
$$

If $f$ is complete it generates a flow as we have seen at Definition 2.1.8. An invariant manifold is a part of the phase space, such that an orbit 2.3.7 inside it never leaves it. More precisely,

Definition 3.0.1 (Invariant manifold). A manifold $S \subset M$ is called invariant under the flow if $\forall t \in \mathbb{R}: \varphi^{t}(S)=S$.

Example 3.0.2. Some cases of invariant manifolds:

- Let $M=\mathbb{R}^{n}$. Fixed point is a single point $x_{0} \in S$ satisfying above condition 3.0.1. In general, the phase space contains more than one fixed point, all isolated. In other words, inside some neighborhood $N_{\delta}\left(x_{0}\right)$ with radius $\delta>0, x_{0}$ is the unique fixed point.

- $S$ is a periodic orbit: there exists a $T>0$ such that $\forall \quad t \in \mathbb{R}: \forall \quad x \in S: \varphi^{t+T}(x)=$ $\varphi^{t}(x)$. The minimum $T$ satisfying this condition it is called period of the cycle.

- Consider $f, g, h: \mathbb{R}^{n} \rightarrow \mathbb{R}^{n}$ and $g(0)=h(0)$ in the system

$$
\dot{x}=f(x)+h(y-x) \quad \dot{y}=f(y)+g(x-y) .
$$

$S=\left\{(x, y) \in \mathbb{R}^{n} \times \mathbb{R}^{n} \mid y=x\right\}$ is an invariant manifold. To prove that $S$ is a manifold, we can use the argument from Chapter 2 that $S$ can be written as the graph of a smooth function $y=F(x) . F \in C^{\infty}$ then $S$ is a $C^{\infty}$ manifold. Moreover, to check that is invariant under the flow of the system, take $\left(x_{0}, y_{0}\right) \in S$. The solution is written as $\varphi^{t}\left(x_{0}, y_{0}\right)$, where $g$ and $h$ are equal due to condition $g(0)=h(0)$ and 
both have the same $f$. So, the vector fields are identical, consequently, $\varphi^{t}\left(x_{0}, y_{0}\right) \in S$ for all time $t$. Looking at $s:=x$ coordinate is a straightforward observation:

$$
\dot{x}=\dot{y}=\dot{s}=f(s)+h(0) \quad \text { which is equal to } g(0)
$$

In view of these previous invariant manifolds examples a question arises: are they stable? In order to answer it we should select the appropriate stability definition. An invariant manifold is stable if a nearby orbit stays close for all time and is unstable otherwise. However, Theorem 3.0.11 requires a stronger definition, because we have to know the rate of convergence of these nearby orbits. This means that we require exponential stability, consisting in whenever we take a nearby solution curve, it converges exponentially fast to a solution lying on the stable invariant manifold — See at Coppel.(20)

Definition 3.0.3 (Exponentially stable). Let $M$ be a metric space. A solution $x(t) \in M$ is exponentially stable if there exists $\delta, C>0$ and $\rho>0$ such that $\forall \varepsilon>0$ there exists $T=T(\epsilon)>0$ such that if $\varphi^{s}\left(y_{0}\right) \in N_{\delta}(x(s))$ for some $s>0$ then:

- $\forall t>T: d\left(\varphi^{t}\left(y_{0}\right), x(t)\right) \leq C e^{-\rho t}$.

Example 3.0.4. • Consider the following autonomous system

$$
\dot{x}=\left(\begin{array}{cc}
-1 & 0 \\
0 & -2
\end{array}\right) x \text {. }
$$

The point $x_{0}=0$ is a fixed point. Any solution is given by $x(t)=e^{A t} x(0)$, where $x(0)$ is the initial condition. Since $A$ is diagonal, it is easy to see that any point goes exponentially to zero. Then $x_{0}$ is exponentially stable.

- The opposite direction concerning synchronous manifold stability is applied as well: in order to be exponentially stable which conditions the coupling function must satisfy? This issue has attracted attention of researchers in last decades. I invite you to read Stankovski et. al. (21) and references therein.

Before we dive into nonlinear differential equations, let us remind some properties of linear setup. Consider system (3.1) where $f$ is linear, so $\dot{x}=A x$ with $A \in \mathcal{L}\left(\mathbb{R}^{n}, \mathbb{R}^{n}\right)$. $\mathbb{R}^{n}$ is decomposed in how $A$ transforms the space, split in three situations: $A$ contracts, expands or rotates the phase space. In terms of generalized eigenvectors of A, it forms a basis in $\mathbb{R}^{n}$. We introduce the notation in Definition 3.0.5 and establish the splitting and exponential estimates in Proposition 3.0.6 — See Teschl (12) and Lancaster. (22) 
Definition 3.0.5 (Invariant spaces). Let the equation $\dot{x}=A x$ with $x \in \mathbb{R}^{n}$ and $A \in$ $\mathcal{L}\left(\mathbb{R}^{n}, \mathbb{R}^{n}\right) . V_{\lambda}=\operatorname{ker}\left(\left(A-\lambda I_{n}\right)^{n}\right)$ denotes the generalized eigenspace of $A$ associated to the eigenvalue $\lambda$ (if $\lambda$ is not an eigenvalue, $V_{\lambda}=\{0\}$ is defined) and

$$
E(I)=\bigoplus_{\operatorname{Re}(\lambda) \in I} V_{\lambda}
$$

is the space associated to all eigenvalues with real part in the interval $I$. We denote the stable, center and unstable spaces as $E^{s}=E(-\infty, 0), E^{c}=E(0)$ and $E^{u}=E(0, \infty)$.

Proposition 3.0.6. Using the Definition 3.0.5 there is the decomposition $\mathbb{R}^{n}=E^{s} \oplus E^{c} \oplus$ $E^{u}$ where $E^{s}, E^{c}$ and $E^{u}$ are invariant under the flow of $A$. There exist $\rho_{s}<0<\rho_{u}$ and $C_{s}, C_{u}>0$ such that the following exponential estimates are valid:

$$
\begin{aligned}
& \forall x \in E^{s}, t \geq 0:\left\|e^{A t} x\right\| \leq C_{s} e^{\rho_{s} t}\|x\|, \\
& \forall x \in E^{u}, t \leq 0:\left\|e^{A t} x\right\| \leq C_{u} e^{\rho_{u} t}\|x\| .
\end{aligned}
$$

For $x \in E^{c}$ there is no exponential rate of convergence, since the eigenvalues are purely imaginary.

Proof. See at Chicone .(11)

With these properties in hand, nonlinear autonomous system close to a fixed point can be analyzed in order to prepare for Theorem 3.0.11. Consider Equation (3.1) and assume that $x_{0}=0$ is a fixed point. The Jacobian matrix $D f\left(x_{0}\right)$ is denoted by $A \in$ $\mathcal{L}\left(\mathbb{R}^{n}, \mathbb{R}^{n}\right)$. The point $x_{0}$ is called hyperbolic if all eigenvalues of $A$ have real part different than zero. For example, the point $x_{0}=(0,0)$ is hyperbolic in the system $(3.2)$.

Hyperbolicity implies that certain qualitative properties of system are unaltered under small perturbations. For instance, invariant manifolds existence around a hyperbolic fixed point, likewise Proposition 3.0.6, is still valid for the nonlinear case. Otherwise these invariant subspaces are "wiggled" a bit. More precisely, Theorem 3.0.8 states that the aforementioned system with a hyperbolic fixed point has stable, center and unstable manifolds $W^{s}\left(x_{0}\right), W^{c}\left(x_{0}\right)$ and $W^{u}\left(x_{0}\right)$, respectively, tangent at $x_{0}$ to the eigenspaces of $A$ : $E^{s}, E^{c}$ and $E^{c}$. Locally ${ }^{1}$ these nonlinear invariant manifold can be characterized as below

Definition 3.0.7 (Local stable/unstable manifold). Let $x_{0}$ be a hyperbolic fixed point of the differential equation (3.1) with $V$ a small neighborhood of $x_{0}$. We say that the local

1 We restrict the manifold $W^{s}\left(x_{0}\right)\left(W^{u}\left(x_{0}\right)\right)$ to a submanifold taking the intersection of a small neighborhood of $x_{0}$, for instance $V$ as we have done at definition 3.0.7. 
stable manifold

$$
W_{l o c}^{s}\left(x_{0}\right):=\left\{x \in V \mid \lim _{t \rightarrow \infty} \varphi^{t}(x)=x_{0}\right\}
$$

and analogously, the local unstable manifold

$$
W_{l o c}^{u}\left(x_{0}\right):=\left\{x \in V \mid \lim _{t \rightarrow-\infty} \varphi^{t}(x)=x_{0}\right\}
$$

The version below was based on Kuznetsov (23) due to the summarized exposition. Theorem 3.0.8 is stated as follows:

Theorem 3.0.8 (Local Stable and Unstable Manifold). Let $x_{0}$ be a hyperbolic and a small neighborhood $V$ containing $x_{0}$. Then there exist $W_{l o c}^{s}\left(x_{0}\right):=V \cap W^{s}\left(x_{0}\right)$ and $W_{l o c}^{u}\left(x_{0}\right):=$ $V \cap W^{u}\left(x_{0}\right)$ tangent at $x_{0}$ to $E^{s}$ and $E^{u}$, respectively.

Proof. See at Chicone (11) for a global version of the theorem.

The proof strategy relies on the Perron method. A space of functions with an appropriate norm is chosen taking careful attention to control both contracting and expanding rates. Afterwards, running a Banach fixed point argument (found at Rudin (24)) for a map involving the flow inside such space, yields a unique manifold written as graph of a function which is invariant by the flow. All this summarized idea is further detailed at Chicone. (11)

Further implications are available when we deal with hyperbolic fixed points. Inside a small neighborhood of the fixed point, the original flow is topologically equivalent to a flow generated by the linearized vector field. There exists an homeomorphism between the original and the linear version. This is called Hartman-Grobman Theorem and can be seen at Chicone (11) and Teschl.(12)

Moreover, results in structural stability is also obtained. It quantifies how much a vector field can be deformed without harming qualitatively the dynamical behavior in a fixed point's small neighborhood. For instance, persistence of fixed points and generic normally hyperbolic invariant manifold are included in this perspective. To begin we need to introduce $C^{k}$-norm of a function $f \in C^{k}(M)$ :

$$
\|f\|_{C^{k}}=\sup _{x \in M} \sum_{l=0}^{k}\left\|f^{l}(x)\right\| .
$$

Mostly we are interested to consider when two vector fields are $C^{1}$-close in respect to the above norm, because in this way, vector field derivatives are controlled close to the 


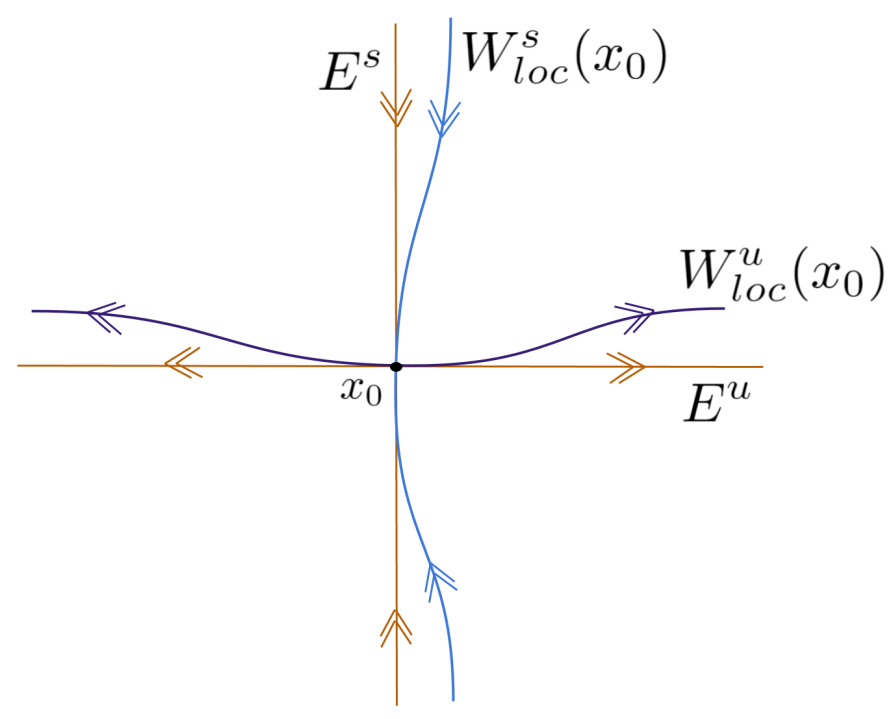

Figure 3 - Stable and unstable linear invariant subspaces $E^{s}$ and $E^{u}$ respectively. $W_{l o c}^{s}\left(x_{0}\right)$ and $W_{l o c}^{u}\left(x_{0}\right)$ represent nonlinear invariant manifold tangent to respective linear spaces at fixed point $x_{0}$.

Source: By the author.

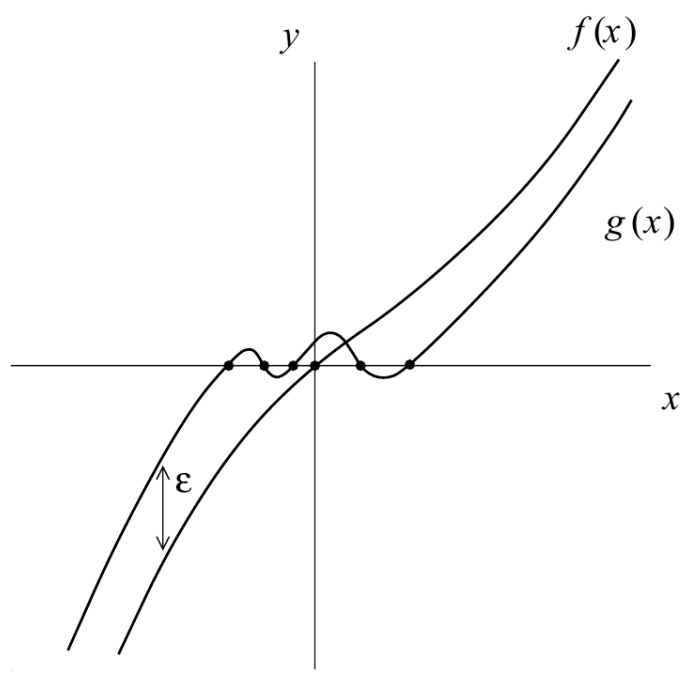

Figure 4 - Two function $f$ and $g$ are $C^{0}$-close. In the plot $f(x)$ has a unique fixed point at $x_{0}=0$. On the other hand, $g(x)$ possesses couple fixed points, spoiling arguments in a small neighborhood of $x_{0}=0$.

Source: KUZNETSOV.(23)

fixed point and similar flows are guaranteed. In contrast, the qualitative phase portrait is not equivalent, see for example two functions $f$ and $g$ that are simply $C^{0}$-close in Figure 4 , they have different number of fixed points.

Regarding persistence of a hyperbolic fixed point against small perturbations, let the system Equation (3.1) be perturbed by a small perturbation: 


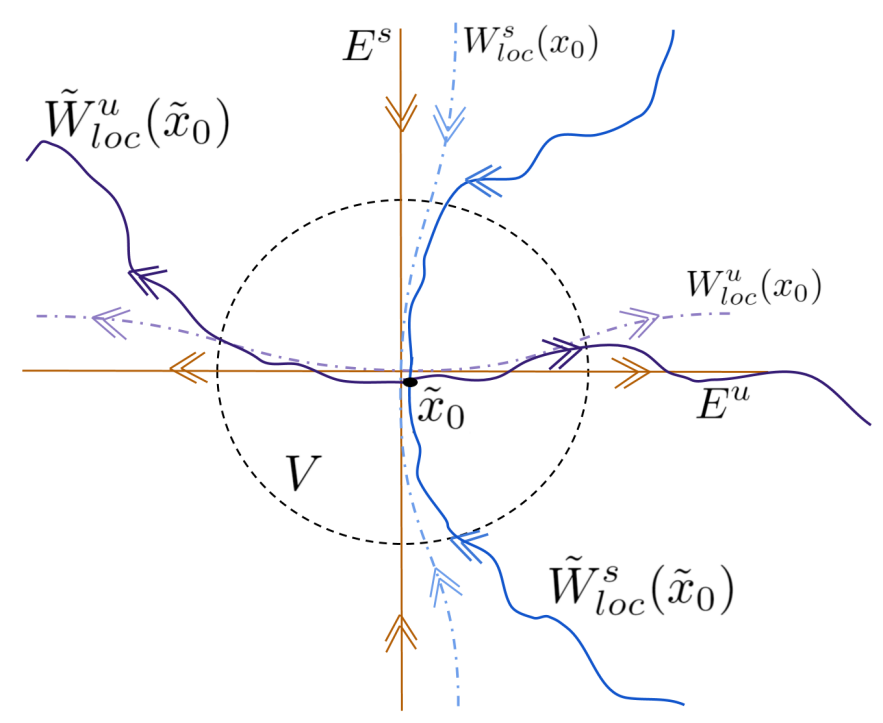

Figure 5 - After a perturbation the system got two new stable and unstable nonlinear invariant manifolds, $\tilde{W}_{l o c}^{s}\left(\tilde{x}_{0}\right)$ and $\tilde{W}_{l o c}^{u}\left(\tilde{x}_{0}\right)$ respectively, at $\tilde{x}_{0}$ which is the perturbed fixed point. Unperturbed invariant manifolds are indicated in dashed-lines. Note that outside neighborhood $V$, they do not coincide or are close anymore.

Source: By the author.

$$
\dot{x}=f(x)+\epsilon g(x)=: \tilde{f}(x), \quad x \in \mathbb{R}^{n} \quad \text { and } f, g \in C^{1} .
$$

If $f$ and $\tilde{f}$ are $C^{1}$-close, i.e. $\|f-\tilde{f}\|_{C^{1}} \leq \epsilon$, then close to $x_{0}$ there is not a qualitative change. This result is consequence of Implicit Function Theorem (found at Rudin (24)). The function $\tilde{f}: \mathbb{R}^{n} \times I \rightarrow \mathbb{R}^{n}$, where $\varepsilon \in I=(-\delta, \delta)$ for small $\delta>0$. Then Implicit Function Theorem assures existence of a function $x(\epsilon)$ such that $\tilde{f}(x(\epsilon), \epsilon)=0$. It preserves the fixed point inside original $x_{0}$ neighborhood although moved slightly. Additionally, $x(\epsilon)$ is still hyperbolic, so Theorem 3.0.8 keeps valid though slightly wiggled, see Figure 5. Look at Wiggins (25) and Kuznetsov (23) for further discussion.

Treating the fixed point case is instructive since the argument to construct normal hyperbolic invariant manifold structure resembles a Implicit Function Theorem generalized to manifolds. See at Fenichel (26) for a historical background.

Finally we define Normally hyperbolic invariant manifold (NHIM, for short):

Definition 3.0.9 (Normally hyperbolic invariant manifold). A submanifold $M \subset \mathbb{R}^{n}$ is a NHIM for the system $f$ if the following hold:

i. $M$ is invariant.

ii. There exists a continuous splitting of the tangent spaces over $M: \forall x \in M: T_{x} \mathbb{R}^{n}=$ $T_{x} M \oplus E_{x}^{s} \oplus E_{x}^{u}$, which is kept invariant by the tangent flow $D \varphi^{t}$. 
iii. There exist real number $a<0<b$ and $C>0$ such that the tangent flow is exponentially contracting/expanding on $E^{s}$ and $E^{u}$ respectively:

$$
\begin{aligned}
& \forall t \geq 0, x \in M, v \in E_{x}^{s}:\left\|D \varphi^{t}(x) v\right\| \leq C e^{a t}\|v\|, \\
& \forall t \leq 0, x \in M, v \in E_{x}^{u}:\left\|D \varphi^{t}(x) v\right\| \leq C e^{b t}\|v\| ;
\end{aligned}
$$

iv. The exponential rates along the normal directions $E^{s}$ and $E^{u}$ dominate any contraction or expansion along the tangent space of each $x \in M$, that is, there exist $r \geq 1, \rho>0$ and $C>0$ such that

$$
\begin{aligned}
& \forall t \geq 0, x \in M:\left\|\left.D \varphi^{t}(x)\right|_{E_{x}^{s}}\right\| \leq C e^{-\rho t} m\left(\left.D \varphi^{t}(x)\right|_{T M}\right)^{r}, \\
& \forall t \leq 0, x \in M:\left\|\left.D \varphi^{t}(x)\right|_{E_{x}^{u}}\right\| \leq C e^{\rho t} m\left(\left.D \varphi^{t}(x)\right|_{T M}\right)^{r},
\end{aligned}
$$

where $m(A)$ is the minimum norm. The minimum norm $m(A)$ of $A \in \mathcal{L}\left(\mathbb{R}^{n}, \mathbb{R}^{n}\right)$ is defined as

$$
m(A)=\inf \{|A x|:|x|=1\} .
$$

When $A$ is invertible, $m(A)=\left\|A^{-1}\right\|^{-1}$.

Each point (ii.), (iii.) and (iv.) can be explained as follows: splitting procedure performed on the linear system is extended for every point $x \in M$, in this occasion, on each tangent space at $x \in M$. Suppose you take a state $x \in \mathbb{R}^{n}$ and flow it by $\varphi^{t}(x)$. The tangent flow (or the linear part) $D \varphi^{t}(x)$ is decomposed in three directions with exponential contraction/expansion rates likewise we have done in 3.0.6. It means, we decompose orbits on the phase space as contracting to $M$ in $E^{s}$-direction, expanding from $M$ in $E^{u}$-direction and staying itself on $M$.

The minimum norm $m\left(D \varphi^{t}(x)\right)$ measures the minimum change that the tangent flow $D \varphi^{t}(x)$ performs to a vector of $T M$. Since the estimates of convergence are calculated by the minimum norm, it guarantees that convergence of normal directions always dominates any contraction or expansion along each tangent space of points in $M$.

Remark 3.0.10. Let $M_{1}, M_{2}$ NHIM's. Then the product $M_{1} \times M_{2}$ is NHIM and the ambient space $\mathbb{R}^{n}$ could be replaced by a smooth manifold $V$.

The main result for NHIM's we use is the Persistence theorem. The theorem extends what we motivated with fixed points. Instead of singular points, there is a whole differential structure defined above which persists against $C^{1}$-close perturbations. Figure 6 depicts a geometrical idea.

Theorem 3.0.11 (Persistence of normally hyperbolic invariant manifolds). Let $M$ be an NHIM for the system $f \in C^{k}$. For any $\tilde{f}$ sufficiently close to $f$ in $C^{1}$-norm, there exists 


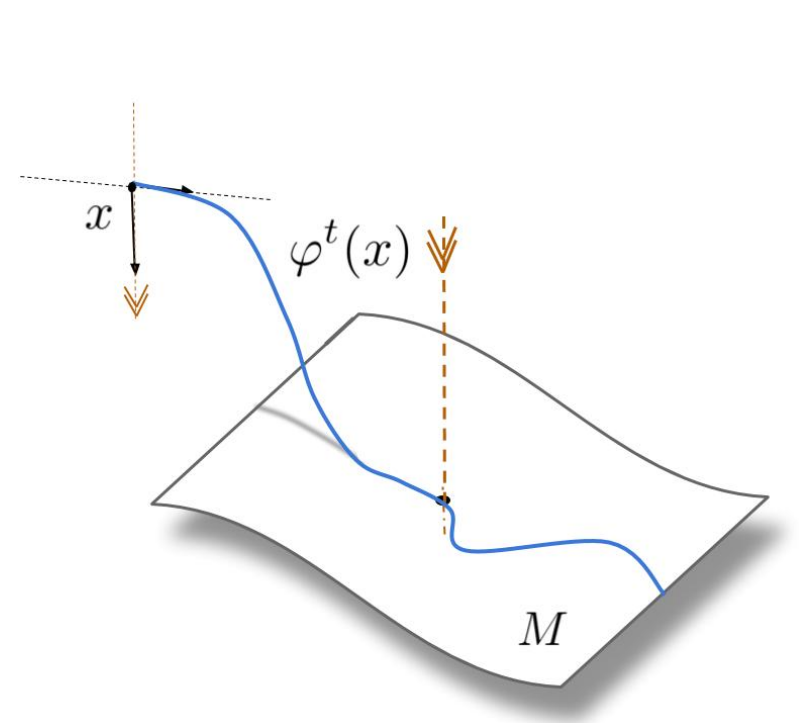

(a) Unperturbed structure

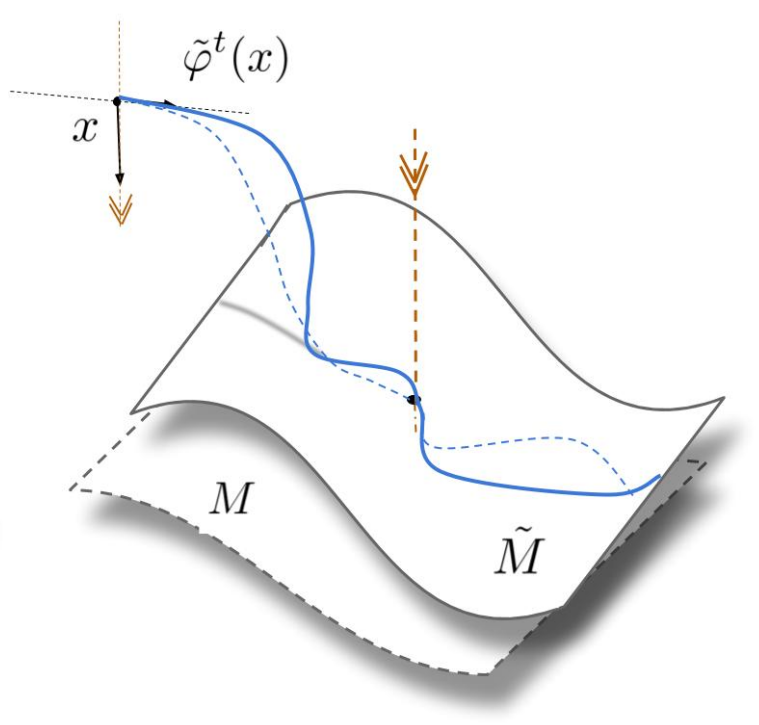

(b) Perturbed structure

Figure 6 - Normal hyperbolicity representation: (a) $M$ is a normally attracting invariant manifold, where there are contracting transverse directions represented in orange dashed lines. Blue solid line consists of a trajectory starting in $x$ and evolved by the flow $\varphi^{t}$. After solution enters $M$, remains there for all time. (b) $\tilde{M}$ is the perturbed version of $M$. The trajectory and the perturbed manifold keep their qualitative properties though wiggled a bit. In contrast, prior structures are dashed-displayed.

Source: By the author.

a unique manifold $\tilde{M}$ that is invariant under $\tilde{f}$ and $C^{1}$-diffeomorphic and close to $M$. We have $\tilde{M} \in C^{k}$ and $\tilde{M}$ is a NHIM again.

Proof. The proof can be found at Fenichel (26) or Wiggins. (25)

\subsection{Characterizing macroscopic states}

The systems of coupled oscillators are identified by solution curves expressed in terms of phases, which we denote $\phi \in S^{1}$. We aim at treating the macroscopic behavior of the system, consisting of a visible observable in the large scales without caring about local dynamics. In other words, the phases' actual value is not important, but the value of a collection of phases. Consequently, we must know how to represent such large scale point of view. We chose a measure called order parameter which makes necessary to define it:

Definition 3.1.1 (Order parameter). Let $N \in \mathbb{N}$ be number of oscillators in the system and $\phi_{k}$ be the phase of the $k$-th oscillator with $k \in\{1, \ldots, N\}$. Let a solution curve 


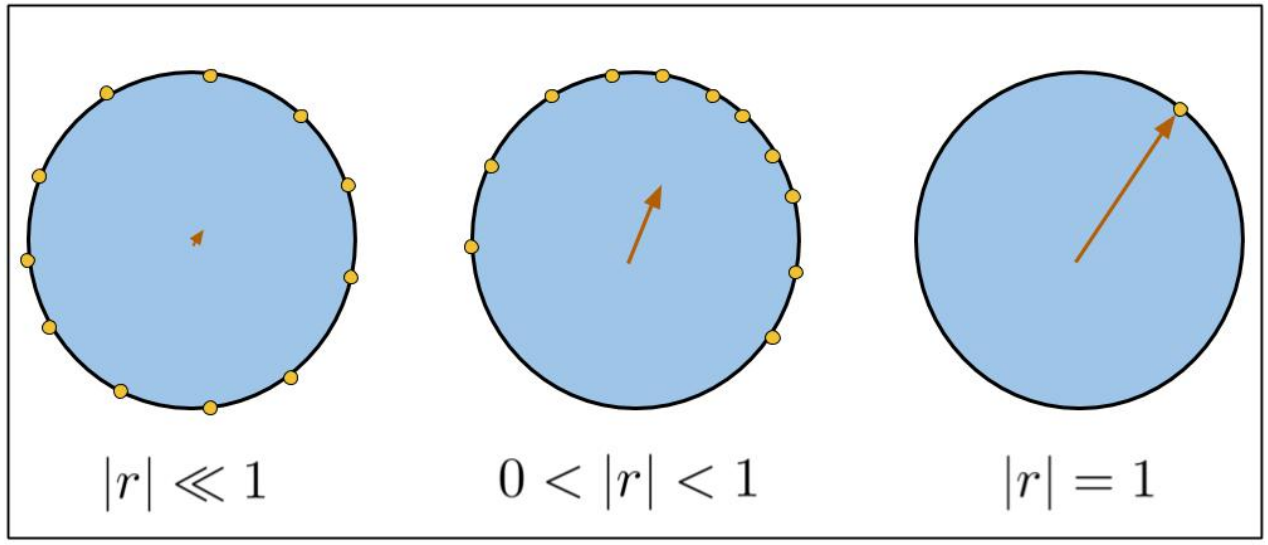

Figure 7 - Order parameter measures phase synchronization. The asynchronous state, identified by the order parameter $|r| \ll 1$, is represented in the left situation. The right plot presents the synchronous state where order parameter measures $|r|=1$.

Source: By the author.

$\gamma: \mathbb{R} \rightarrow \mathbb{T}^{N}$. We define

$$
\begin{aligned}
& r: \mathbb{T}^{N} \rightarrow \mathbb{C} \\
& r(\gamma(t))=\frac{1}{N} \sum_{l=1}^{N} e^{i \phi_{l}(t)} .
\end{aligned}
$$

We introduced the order parameter as a complex-valued measure. In the literature, an alternative definition is found where $|r|$ is named order parameter and $\arg (r)$ is the average phase. We choose the complex version since the concept is suppressed to an unique parameter. Figure 7 displays a common geometric representation. We observe that order parameter captures the large macroscopic states by its absolute value. By the triangular inequality in expression (3.11) the order parameter modulus is limited $|r| \leq 1$. So, we are able to recognize two situations: when the modulus is approximately one; and on contrary, close to zero. Let us be more precise

Definition 3.1.2 (Synchronous and Asynchronous states). Consider a system which admits order parameter $r$ (definition 3.1.1). Given a small $\sigma>0$ there exists

- Synchronous state: if $1-\sigma<|r(t)| \leq 1$.

- Asynchronous state: if $|r(t)|<\sigma$,

for $t$ large enough.

Both states are dependent of the same precision $\sigma$ you choose and are uniform in time. Returning to Figure 7, the left situation is an asynchronous state; and the right is 
a synchronous one, which hold for a given small $\sigma$. The middle situation is ambiguous, so we will not cover it for the moment.

Note that synchronous state is realized when order parameter assumes close to maximum value. Looking at Equation (3.11) configuration and Definition 3.1.2, it occurs when all phases are nearby each other; which in the extreme situation, when they are all equal. Therefore, let us recapitulate Example 3.0.2 on identical units. A set $S$ with equal coordinates for the states defined a smooth manifold, since it was a graph of the identity function. This illustrates a precise way of connecting macroscopic states with manifold structures on phase space considering the extreme situation: a synchronous or coherent state constitute for all phases are equal.

Remark 3.1.3 (Phase locking). Even for nonidentical oscillators system, the coherent state is achieved. Differently from identical oscillators, the phase difference among oscillators are constant instead of the phase itself. We say they are phase locked. The system we proved our result (1.1) covers this case.

Splay-states. On the contrary direction, asynchronous state corresponds to minimum order parameter values. The limit case of zero value represents phases spread apart on the unite circle, which receives a special name splay-state. Let us analyze it.

Definition 3.1.4 (Splay-state). Let $N$ be the number of oscillators and for $k \in\{1, \ldots, N\}$ let $\theta_{k} \in S^{1}$ be the phase of the $k$-oscillator. We say that the collection of $\theta^{\prime}$ s forms a splay state if there exists $\xi \in S^{1}$ such that $\theta_{k}=\frac{2 \pi k}{N}+\xi$ up to a permutation.

Lemma 3.1.5 proves what we mentioned before without being precise. Splay-state has the minimum value of order parameter 3.11:

Lemma 3.1.5. Let $N \in \mathbb{N}$ the number of oscillators, $n \in \mathbb{N} \backslash\{0, N\}$ and $k \in\{1, \ldots, N\}$. If a collection of oscillators is in a splay state then $\sum_{k=1}^{N} e^{i n \theta_{k}}=0$, where $\theta_{k}$ is the $k$ oscillator phase. In particular, the order parameter corresponds to the case $n=1$.

Proof. Take $\theta_{k}=\frac{2 \pi k}{N}+\xi$, then

$$
\begin{aligned}
\sum_{k=1}^{N} e^{i n \theta_{k}} & =\sum_{k=1}^{N} e^{i n \xi} e^{i \frac{2 \pi n k}{N}} \\
& =e^{i n \xi}\left[\sum_{k=1}^{N}\left(e^{i \frac{2 \pi n}{N}}\right)^{k}\right] \\
& =\left[\frac{1-e^{i 2 \pi n}}{1-e^{i \frac{2 \pi n}{N}}}\right] \\
& =0,
\end{aligned}
$$


for any $n \neq N$, where we used the result of partial sum of a geometric series.

These splay-states play key role to the Möbius group reduction method. See at Chapter 4 .

Synchronization transitions. Note that order parameter definition 3.1.1 it only has a single value when a system state is plugged in. However, the system may depend on parameters, namely the coupling strength. Varying coupling strength, macroscopic state alters. Consider macroscopic state definition 3.1.2. Given a $\sigma$ small enough, synchronization transition occurs when the macroscopic state changes after gradual variation of coupling among the oscillators. In particular if the system starts from a splay-state and one increases coupling strength in small steps, order parameter magnitude may violate $\sigma$ precision, and consequently incoherent state is lost and collective behavior starts to emerge. A key feature of this phenomenon is robustness since similar scenario will happen if the system starts close to splay-state.

The opposite direction is valid as well. For decreasing coupling, the order parameter magnitude decreases. After it passes over the $\sigma$ precision at threshold coupling, the synchronous state ceases. We say there exists a discontinuous transitions if for given system parameters, there can be two different stable states where the order parameter takes two different values at the critical parameter value and hysteresis is present. Otherwise, there is a continuous transition. From here on, we will consider only variation on coupling strength. 



\section{Identical coupled oscillators}

In this chapter Möbius group reduction method is presented via a Theorem 4.1.1 form.

\subsection{Möbius group reduction}

Consider a class (or family) of oscillators which are described by the following system

$$
\dot{\phi}_{j}=f e^{i \phi_{j}}+g+\bar{f} e^{-i \phi_{j}}, \quad j=1, \ldots, N
$$

where $\phi_{j} \in S^{1}$ is the $j$ th phase oscillator, $f: \mathbb{R} \times \mathbb{T}^{N} \rightarrow \mathbb{C}$ and $g: \mathbb{R} \times \mathbb{T}^{N} \rightarrow \mathbb{R}$ are smooth functions with time $t \in \mathbb{R}$ and $\left(\phi_{1}, \ldots, \phi_{N}\right) \in \mathbb{T}^{N}$. If the functions $f$ and $g$ are time independent, we drop such time dependence.

Let $\mathbb{D}=\{z \in \mathbb{C}:|z|<1\}, \alpha \in \mathbb{D}, \psi \in S^{1}, \forall j=1, \ldots, N: \theta_{j} \in S^{1}$ constants in time. The Möbius transformation $\mathcal{F}_{\alpha, \psi}$ is

$$
\mathcal{F}_{\alpha, \psi}\left(e^{i \theta_{j}}\right)=\frac{e^{i \psi}\left(e^{i \theta_{j}}\right)+\alpha}{1+\bar{\alpha} e^{i \psi}\left(e^{i \theta_{j}}\right)},
$$

which is the element of the automorphism of the unit disc, Aut $\mathbb{D}$. We define the mapping $\pi: \mathbb{D} \times S^{1} \times \mathbb{T}^{N} \rightarrow \mathbb{T}^{N}$ to be an $n$-tuple of the Möbius group diagonal action with each component corresponding to

$$
e^{i \phi_{j}}=\mathcal{F}_{\alpha, \psi}\left(e^{i \theta_{j}}\right), \quad j=1, \ldots, N
$$

With this in hand, the Theorem 4.1.1 is stated as follows:

Theorem 4.1.1 (Möbius group reduction). Consider $N$ identical oscillators described by 4.1. Let $\mathbb{D}=\{z \in \mathbb{C}:|z|<1\}, \alpha \in \mathbb{D}, \psi \in S^{1}, \forall j=1, \ldots, N: \theta_{j} \in S^{1}$ constants in time. The solution curves of the following system

$$
\begin{aligned}
& \dot{\alpha}=i\left(f \alpha^{2}+g \alpha+\bar{f}\right) \\
& \dot{\psi}=f \alpha+g+\bar{f} \bar{\alpha} \\
& \dot{\theta}_{j}=0 \quad j=1, \ldots, N .
\end{aligned}
$$


maps to solution curves of Equation (4.1) if we apply the mapping $\pi$ of Equation (4.3).

Moreover, let $z=\left(z_{1}, \ldots, z_{N}\right) \in \mathbb{C}^{N}$ with $\forall j=1, \ldots, N: z_{j}=e^{i \phi_{j}}, \phi_{j} \in S^{1}$. The system (4.1) conserves $N-3$ constants of motion given by independent cross-ratios $C_{\text {pqrs }}(z)$ of four distinct coordinates $z_{p}, z_{q}, z_{r}, z_{s}$ of the $N$-tuple $z$ for all $p, q, r, s$ in $\{1, \ldots, N\}$ expressed by:

$$
C_{p q r s}(z)=\frac{\left(z_{p}-z_{r}\right)\left(z_{q}-z_{s}\right)}{\left(z_{p}-z_{s}\right)\left(z_{q}-z_{r}\right)}
$$

Proof. Proof 1: In order to prove theorem 4.1.1, we divide in two parts proving on one hand the change of coordinates proposed, and on the other hand, cross ratios as constants of motion.

First let us prove that cross-ratios are constants under the dynamics given by equation (4.1) following Stewart (19) steps. Let $\forall j=1, \ldots, N: z_{j}=e^{i \phi_{j}}, \phi_{j} \in S^{1}$, then the differential equation (4.1) is equivalent to equation (4.6).

$$
\dot{z}_{j}=i\left(f z_{j}^{2}+g z_{j}+\bar{f}\right), \quad j=1, \ldots, N
$$

We assume that $\forall j, l=1, \ldots, N: z_{j} \neq z_{l}$, because cross-ratio is defined for fourdistinct points. In order to analyze the time dependence of cross ratios functions, we calculate first their logarithm to facilitate some ponderous manipulation calculus. Then we obtain equation (4.7).

$$
\log \left(\lambda_{\text {pqrs }}(z)\right)=\log \left(z_{p}-z_{r}\right)+\log \left(z_{q}-z_{s}\right)-\log \left(z_{p}-z_{s}\right)-\log \left(z_{q}-z_{r}\right)
$$

Taking the time derivative of expression (4.7) yields:

$$
\frac{d}{d t}\left[\log \left(\lambda_{p q r s}(z)\right)\right]=\frac{\dot{z_{p}}-\dot{z}_{r}}{\left(z_{p}-z_{r}\right)}+\frac{\dot{z}_{q}-\dot{z}_{s}}{\left(z_{q}-z_{s}\right)}-\frac{\dot{z_{p}}-\dot{z}_{s}}{\left(z_{p}-z_{s}\right)}-\frac{\dot{z}_{q}-\dot{z}_{r}}{\left(z_{q}-z_{r}\right)} .
$$

Each component $z_{i}$ is governed by the equation 4.6, then we replace each correspondent coordinate at cross ratios functions by the respectively differential equation. Consider the first term at right hand side:

$$
\frac{\dot{z}_{p}-\dot{z}_{r}}{\left(z_{p}-z_{r}\right)}=\frac{i\left[f\left(z_{p}^{2}-z_{r}^{2}\right)+g\left(z_{p}-z_{r}\right)\right]}{\left(z_{p}-z_{r}\right)}=i f\left(z_{p}+z_{r}\right)+g
$$


Repeating such manipulation for every term at the right hand side in equation (4.8) we obtain the following:

$\frac{d}{d t}\left[\log \left(\lambda_{p q r s}(z)\right)\right]=i f\left(z_{p}+z_{r}\right)+g+i f\left(z_{q}+z_{s}\right)+g-\left[i f\left(z_{p}+z_{s}\right)+g\right]-\left[i f\left(z_{q}+z_{r}\right)+g\right]=0$.

Then we conclude that the cross ratio functions are constants over time, or in more common terminology, constants of motion. ${ }^{1}$

In order to prove the first part of the theorem we must take the transformation $\pi$ in (4.3). This yields:

$$
\begin{aligned}
& e^{i \phi_{j}}=\mathcal{F}_{\alpha, \psi}\left(e^{i \theta_{j}}\right) \\
& i e^{i \phi_{j}} \dot{\phi}_{j}=\frac{d}{d t}\left[\mathcal{F}_{\alpha, \psi}\left(e^{i \theta_{j}}\right)\right] \\
& i \mathcal{F}_{\alpha, \psi}\left(e^{i \theta_{j}}\right)\left[f \mathcal{F}_{\alpha, \psi}\left(e^{i \theta_{j}}\right)+g+\bar{f} \frac{1}{\mathcal{F}_{\alpha, \psi}\left(e^{i \theta_{j}}\right)}\right]=\frac{d}{d t}\left[\mathcal{F}_{\alpha, \psi}\left(e^{i \theta_{j}}\right)\right] .
\end{aligned}
$$

After algebraic calculus where the term $\mathcal{F}_{\alpha, \psi}\left(e^{i \theta_{j}}\right)$ is replaced by equation (4.2) (see Marvel, Mirollo and Strogatz (15) - at section III.A), we are able to obtain explicitly the differential equation for $\alpha$ and $\psi$ :

$$
\begin{array}{ll}
\dot{\alpha}=i\left(f \alpha^{2}+g \alpha+\bar{f}\right), & \\
\dot{\psi}=f \alpha+g+\bar{f} \bar{\alpha} & \\
\dot{\theta}_{j}=0, & j=1, \ldots, N .
\end{array}
$$

Proof 2: The proof relies on the fact mentioned at end of Section 2.3, Theorem 2.3.8 and the Fundamental Theorem of Vessiot-Guldberg-Lie 2.3.11.

Consider $\forall j=1, \ldots, N: z_{j}=e^{i \phi_{j}} \in \partial \mathbb{D}$, then the differential equation (4.1) is equivalent to equation (4.6) which consists of a $N$-dimensional Riccati equation. Riccati equation, as we have seen in Chapter 2 , generates the Möbius group action on $\hat{\mathbb{C}}$. So, the solutions of Equation (4.1) lie on group orbits of Möbius group.

Each $z_{j}$ lies on the boundary on the unit disc, then the Möbius group action must preserve the unit disc and consequently its boundary. Therefore the elements $\mathcal{F}_{\alpha, \psi}$ of the Möbius group take the form as in Equation (4.2). The group orbit is identified for each

1 We are allowed to classify such as constants of motion because the cross ratio do not change over time when solution curves of the equations of motion 4.6 are plugged in it. 
time t by the parameters $\alpha$ and $\psi$ which evolve in time by the Equation (4.16). The crossratios are constants of motion because Möbius group preserve them. The interested reader that desires to see the proof in terms of homogeneous coordinates see at Stewart .(19)

To complement the initial discussion at Chapter 1, note that these particular solutions $\alpha$ and $\psi$ are equal for all $N$ oscillators, since $f$ and $g$ do not depend on the index $j$. This indicates mathematically what we have only argued in the physical perspective: global sinusoidal coupling allows a low-dimensional description. Low-dimensional because $\theta$ 's have trivial dynamics, then effectively the system is only three-dimensional.

\subsection{Order parameter description}

We aim at obtaining the order parameter differential equation and analyze its asymptotic behavior. For this purpose, we must study how to apply the Möbius group reduction theorem. Let $\varphi^{t}: \mathbb{D} \times S^{1} \times \mathbb{T}^{N} \rightarrow \mathbb{D} \times S^{1} \times \mathbb{T}^{N}$ be the flow of the Equation (4.4). If we compose this flow with the mapping $\pi$ of Equation (4.3) for each time $t \in \mathbb{R}$, the order parameter will be regarding initial conditions $\left(\alpha_{0}, \psi_{0}\right)$ and constants $\theta_{l}$ for $l \in\{1, \ldots, N\}$ :

$$
\begin{aligned}
& r\left(\pi \circ \varphi^{t}\left(\alpha_{0}, \psi_{0}, \theta_{1}, \ldots, \theta_{N}\right)\right)=\frac{1}{N} \sum_{l=1}^{N} e^{i \phi_{l}(t)} \\
& r(t)=\frac{1}{N} \sum_{l=1}^{N} \mathcal{F}_{\alpha(t), \psi(t)}\left(e^{i \theta_{l}}\right),
\end{aligned}
$$

where $\alpha(t)$ and $\psi(t)$ are the solution curves.

Note that above expression is not straightforward to solve for any solution curve $\gamma$ of the system $\left(\alpha, \psi, \theta_{1}, \ldots, \theta_{N}\right)$ in Equation (4.4), once involves a nonlinear transformation inside the sum argument in Equation (4.17). So, in the general setup we would fail to give a order parameter description. For this reason, we have to include a new assumption selecting a special choice for the thetas, because they are constant for any time.

For the infinite problem the literature indicates that uniformly distribute thetas on the unit circle yield order parameter evolution similar to the evolution of $\alpha$, see Pikovsky and Rosenblum (27) and Marvel, Mirollo and Strogatz (15). We employ same procedure here, in view of distributing thetas in splay-state configuration 3.1.4 introduces a closed way to calculate the sum term in Equation (4.17). Replace splay-state condition over thetas in Equation (4.17) (we drop time dependence for awhile in order to clarify the calculation): 


$$
\begin{aligned}
r & =\frac{1}{N} \sum_{l=1}^{N} \mathcal{F}_{\alpha, \psi}\left(e^{i \theta_{l}}\right) \\
& =\frac{1}{N} \sum_{l=1}^{N}\left[\frac{e^{i\left(\psi+\frac{2 \pi l}{N}+\xi\right)}+\alpha}{1+\bar{\alpha} e^{i\left(\psi+\frac{2 \pi l}{N}+\xi\right)}}\right] \\
& =\frac{1}{N} \sum_{l=1}^{N}\left(e^{i\left(\psi+\frac{2 \pi l}{N}+\xi\right)}+\alpha\right)\left[\sum_{n=0}^{\infty}(-\bar{\alpha})^{n} e^{i n\left(\psi+\frac{2 \pi l}{N}+\xi\right)}\right] \\
& =\sum_{n=0}^{\infty}\left[(-\bar{\alpha})^{n} \sum_{l=1}^{N} e^{i(n+1)\left(\psi+\frac{2 \pi l}{N}+\xi\right)}+\alpha(-\bar{\alpha})^{n} \sum_{l=1}^{N} e^{i n\left(\psi+\frac{2 \pi l}{N}+\xi\right)}\right] \\
& =\alpha+(-\bar{\alpha})^{N-1} e^{i N(\psi+\xi)}\left(1-|\alpha|^{2}\right),
\end{aligned}
$$

where we expanded in absolutely convergent geometric series since $\alpha \in \mathbb{D}$ and used Lemma 3.1.5. Order parameter equation differs from $\alpha$ 's one and does depends on $\psi$, yielding different result from the infinite setup. This shows an issue we must deal with when treating finite system. Eldering and collaborators handled this issue proving Lemma 4.2.1. It shows that order parameter equation is $C^{k}$-close to $\alpha$ 's. Here I am reproducing results and calculations from their paper. We denote the set of all thetas corresponding to a splay-state by $\Theta \in \mathbb{T}^{N}$.

Lemma 4.2.1. Fix $0<\delta<1$ and let the disc $\mathbb{D}_{\delta}=\{\alpha \in \mathbb{C}|| \alpha \mid<\delta\}$. For each $k \geq 1$ and $\varepsilon>0$ there exists for all $N$ sufficiently large an open neighborhood $U$ of $\Theta \subset \mathbb{T}^{N}$ such that for $\alpha \in \mathbb{D}_{\delta}$ the map $\Delta^{\alpha}$ taking $\alpha \mapsto r\left(\alpha, \psi, \theta_{1}, \ldots, \theta_{N}\right)-\alpha$ it holds

$$
\left\|\Delta^{\alpha}\right\|_{C^{k}} \leq \varepsilon
$$

Proof. Fix a realization of splay state for $\Theta \in \mathbb{T}^{N}$ and consider $l \in\{0, \ldots, N-1\}$ in $\Theta=\left(\theta_{0}, \ldots, \theta_{l}, \ldots, \theta_{N-1}\right)$.

$$
\begin{aligned}
r\left(\alpha, \psi, \theta_{1}, \ldots, \theta_{N}\right) & =\left[\frac{1}{N} \sum_{l=0}^{N-1}\left(\alpha+e^{i\left(\frac{2 \pi l}{N}+\xi+\psi\right)}\right)\right] \sum_{n=0}^{\infty}-\bar{\alpha}^{n} e^{i n\left(\frac{2 \pi l}{N}+\xi+\psi\right)} . \\
& =\left[\frac{1}{N} \sum_{l=0}^{N-1} \alpha\left(1+\alpha^{-1} e^{i\left(\frac{2 \pi l}{N}+\xi+\psi\right)}\right)\right]\left[\sum_{n=0}^{N-2}(-\bar{\alpha})^{n} e^{i n\left(\frac{2 \pi l}{N}+\xi+\psi\right)}+R_{N-2, l}(\alpha)\right] \\
& =\frac{\alpha}{N} \sum_{n=0}^{N-2} \sum_{l=0}^{N-1}\left[\left(-\bar{\alpha}^{n}\right) e^{i n\left(\frac{2 \pi l}{N}+\xi+\psi\right)}+\frac{(-\bar{\alpha})^{n}}{\alpha} e^{i(n+1)\left(\frac{2 \pi l}{N}+\xi+\psi\right)}\right] \\
& =\frac{\alpha}{N} \sum_{n=0}^{N-2} \delta_{n, 0} \sum_{l=0}^{N-1}(-\bar{\alpha})^{n} e^{i n\left(\frac{2 \pi l}{N}+\xi+\psi\right)}+\frac{\alpha}{N}\left(1+\alpha^{-1} e^{i\left(\frac{2 \pi l}{N}+\xi+\psi\right)}\right) R_{N-2, l}(\alpha)
\end{aligned}
$$




$$
\alpha+\frac{1}{N} \sum_{l=0}^{N-1}\left(\alpha+e^{i\left(\frac{2 \pi l}{N}+\xi+\psi\right)}\right) R_{N-2, l}(\alpha),
$$

where $\delta_{i j}$ is the Kronecker delta. The Kronecker delta appears because for $n \in$ $\mathbb{N} \backslash\{0, N-1\}$ the sum from 0 to $N-1$ of $e^{i n\left(\frac{2 \pi l}{N}\right)}$ is zero, look at Lemma 3.1.5.

The second term must be estimated in $C^{k}$-norm. We have

$$
R_{N-2, l}(\alpha)=\sum_{n \geq N-1}\left(-\bar{\alpha} e^{i\left(\frac{2 \pi l}{N}+\xi+\psi\right)}\right)^{n}=(-\bar{\alpha})^{N-1} \sum_{n \geq 0}(-\alpha)^{n}\left(e^{i\left(\frac{2 \pi l}{N}+\xi+\psi\right)}\right)^{n+N-1}
$$

where the sum still defines a power series with radius of convergence one, then $R_{N-2, l}(\alpha)$ is a function in $\mathbb{D}_{r}$. Therefore, it enables to uniformly bound for $\alpha \in D_{\delta}$ the function and its derivatives up to order $k$ by some number $B_{k}>0$

$$
\left\|R_{N-2, l}\right\|_{C^{k}} \leq k \delta^{N-1} B_{k}
$$

Multiplying $R_{N-2, l}(\alpha)$ there is $F_{l}(\alpha)=\alpha+e^{i\left(\frac{2 \pi l}{N}+\xi+\psi\right)}$ bounded in $C^{1}$-norm by $2+\delta$ and all higher derivatives zero. So the product is bounded as below

$$
\begin{aligned}
\left\|\frac{1}{N} \sum_{l=0}^{N-1} F_{l} \cdot R_{N-2, l}\right\|_{C^{k}} & \leq \max _{l \in[0, N-1]}\left\{\left\|F_{l}\right\|_{C^{0}}\left\|R_{N-2, l}\right\|_{C^{k}}+k\left\|F_{l}\right\|_{C^{1}}\left\|R_{N-2, l}\right\|_{C^{k-1}}\right\} \\
& \leq k^{2}(2+r) r^{N-1} B_{k}
\end{aligned}
$$

Therefore, for a fixed $r<1$ and $k \geq 1$ and given $\varepsilon>0$, there exists an $N_{0}>0$ such that for all $N \geq N_{0}$

$$
\left\|\Delta^{\alpha}\right\|_{C^{k}} \leq \varepsilon / 2 \quad \text { on } \mathbb{D}_{\delta} \times S^{1} \times \Theta
$$

After, since this function $\Delta^{\alpha}$ is $C^{\infty}$ (actually analytic with respect to $\alpha, \bar{\alpha}, \psi$, $\left.\left(\theta_{1}, \ldots, \theta_{N}\right)\right)$, it follows that there exists an open neighborhood of $\Theta \subset U$ :

$$
\left\|\Delta^{\alpha}\right\|_{C^{k}} \leq \varepsilon \quad \text { on } \mathbb{D}_{\delta} \times S^{1} \times \Theta,
$$

which completes the proof.

The conclusion from Lemma 4.2.1 is once the order parameter $r$ and $\alpha$ are $C^{k}$ close on $\mathbb{D}_{\delta} \times S^{1} \times U$, they respect closely the same differential equation. As we aforementioned it turns out order parameter equation is obtained from a small perturbation $\alpha$ equation, one of the steps performed to prove our main result in next Chapter 5. 


\section{Statement of the Main Theorem and proof}

With the theory presented in previous chapters we can present the proof of discontinuous transition phenomenon in a star graph. Consider the star system (5.1) with $N$ leaves (peripheral oscillators) coupled with one central oscillator (hub) given by

$$
\begin{aligned}
& \dot{\vartheta}_{k}=\omega+K \sin \left(\vartheta_{H}-\vartheta_{k}+v\right) \quad k=1, \ldots, N, \\
& \dot{\vartheta}_{H}=\beta \omega+\beta \frac{K}{N} \sum_{j=1}^{N} \sin \left(\vartheta_{j}-\vartheta_{H}+v\right),
\end{aligned}
$$

where $\vartheta_{k} \in S^{1}$ is the phase of the $k$-leaf oscillator, $\vartheta_{H} \in S^{1}$ is the phase of the hub oscillator, $0<\omega \in \mathbb{R}$ is the natural frequency, $\beta \in(1, \infty) \subset \mathbb{R}$ is the correlation parameter, $v \in S^{1}$ is the frustration term and $K \in \mathbb{R}$ is the coupling strength. Before proving Theorem 5.0.1, we must identify what are the macroscopic states accessible by the system (5.1). As we have seen these states may characterize invariant manifolds.

In Section 3.1, the synchronous state was presented and defined in terms of the order parameter. We must analyze if we are able to define a manifold associated to it. The leaves and hubs have different vector fields, so it turns out not to be completely identical. If we take the phase difference as new coordinates yields

$$
\begin{gathered}
\phi_{k}=\vartheta_{k}-\vartheta_{H} \quad k=1, \ldots, N \\
\dot{\phi}_{k}=(1-\beta) \omega-K \sin \left(\phi_{k}-v\right)-\beta \frac{K}{N} \sum_{j=1}^{N} \sin \left(\phi_{j}+v\right) \\
\dot{\vartheta}_{H}=\beta \omega+\beta \frac{K}{N} \sum_{j=1}^{N} \sin \left(\phi_{j}+v\right) .
\end{gathered}
$$

The synchronous state is identified by the leaves with $\vartheta$ phase and constant phase difference $\phi$ with the hub. A phase locking case. With this in hand, we define a phase locking manifold

$$
M_{\phi}:=\left\{\vartheta_{1}=\cdots=\vartheta_{N}=\vartheta \in S^{1} \text { and } \vartheta-\vartheta_{H}=\phi \in S^{1}\right\}
$$

where $\phi$ is a parameter. $M_{\phi} \subset \mathbb{T}^{N+1}$ is a smooth one-dimensional manifold. The phase locking manifold in Equation (5.3) yields order parameter equal to one. Nevertheless, 
it is not invariant by the flow of Equation (5.2). For this reason we need Proposition 5.0.3, where we show that there exist specific values of $\phi$ such that $M_{\phi}$ is invariant and $\phi$ is fixed point, denoted by $M_{\text {sync }} \subset \mathbb{T}^{N}$. Moreover, actually we prove that $M_{\text {sync }}$ is normally attracting invariant manifold if coupling $K$ is larger than a positive number $K_{c}^{b}$ - backward critical coupling. Since $M_{\text {sync }}$ is attracting, there exists a open set $\mathcal{V} \subset \mathbb{T}^{N}$ such that initial conditions starting inside it converge to the manifold after long term.

In parallel, asynchronous manifold $M_{\text {async }}$ is defined as well, but we must work on the following issue: the Möbius group theorem can be applied in view of the shape of (5.2). The phases $\phi_{j}$ in Equation (5.2) are written in terms of the Möbius group action when we use the mapping $\pi$ introduced in Equation (4.3). If we choose particular initial $\theta$ phases forming a roughly splay state (see definition at 3.1.4) on $S^{1}$ and the number of leaves $N$ sufficiently large (like a thermodynamic limit), under these conditions, Lemma 4.2.1 presented in last Chapter 4 ensures: $\alpha$ differential equation is $C^{k}$-close to order parameter $r$. Studying Equation for $\alpha$ in Proposition 5.0.4 we define an asynchronous manifold in $\mathbb{D} \times S^{1}$, for coupling $K$ less than a positive number called forward critical coupling $K_{c}^{f}$.

In addition, Lemma 5.0.7 runs a persistence argument, since $\alpha$ and $r$ are $C^{k}$-close. The asynchronous manifold in $\mathbb{D} \times S^{1}$ is extended to an open set $U$ of $\theta$ s close to the splay state determining the $M_{\text {async }} \subset \mathbb{D} \times S^{1} \times \mathbb{T}^{N}$. For fixed $\alpha, \psi$ the mapping $\pi$ is a diffeomorphism then maps open set $U$ to $\mathcal{U} \in \mathbb{T}^{N}$. If the initial conditions are chosen inside the open set $\mathcal{U}$ then the order parameter will be close to zero after long time.

The last step is showing that given a small $\sigma>0$ there exists a $\beta_{0}$ sufficiently large such that after $K_{c}^{f}$ order parameter goes from $\sigma$ to close to one abruptly. And in opposite direction as well, considering $K_{c}^{b}$.

Our main Theorem is stated:

Theorem 5.0.1 (Main theorem). Consider the star system (5.2). Let $\omega>0, v \in(0, \pi / 4)$ and $\varphi^{t}$ be the flow of Equation (5.2). Given a $\sigma>0$ there exists $N_{0}>0$ and $\beta_{0}>0$ such that for each $N>N_{0}, \beta>\beta_{0}$ and $K>K_{c}^{b}$ there exists an open set $\mathcal{V} \subset \mathbb{T}^{N}$ such that

$$
\text { if }\left(\phi_{1}^{0}, \ldots, \phi_{N}^{0}\right) \in \mathcal{V} \quad \text { then }\left|r\left(\varphi^{t}\left(\phi_{1}^{0}, \ldots, \phi_{N}^{0}\right)\right)\right|>1-\sigma \quad \forall t \in \mathbb{R} .
$$

Moreover, there exists for each $0<K<K_{c}^{f}$ an open set $\mathcal{U} \subset \mathbb{T}^{N}$ such that

$$
\text { if }\left(\phi_{1}^{0}, \ldots, \phi_{N}^{0}\right) \in \mathcal{U} \text { then }\left|r\left(\varphi^{t}\left(\phi_{1}^{0}, \ldots, \phi_{N}^{0}\right)\right)\right|<\sigma \quad \forall t \in \mathbb{R} \text {. }
$$

\subsubsection{Synchronous manifold}

Proposition 5.0.3 states that there is a given interval of coupling strength and a $\phi \in S^{1}$ in $M_{\text {sync }}(\phi)$ expression 5.3 such that synchronous manifold is normally attracting 
invariant manifold. First we need to have the following

Hypothesis 5.0.2. Let $\omega>0, \beta \in(1, \infty)$. Assume $K>0$ such that there exists a $v \in S^{1}$ which defines $\mathbf{v}=(\beta \sin (2 v), 1+\beta \cos (2 v))$. The following must hold

$$
\arg (\mathbf{v})-\arccos \left(\frac{(\beta-1) \omega}{\|\mathbf{v}\| K}\right) \in(-\pi / 2, \pi / 2)
$$

where $\|\cdot\|$ denotes the standard Euclidean intern product.

Proposition 5.0.3. Let and $\mathbf{v}=(\beta \sin (2 v), 1+\beta \cos (2 v))$. $M_{\text {sync }}$ is normally attracting invariant manifold if and only if $K>K_{c}^{b}:=\frac{(\beta-1) \omega}{\|\mathbf{v}\|}$ and

$$
\zeta=v-\pi+\arctan \left(\frac{1+\beta \cos (2 v)}{\beta \sin (2 v)}\right)+\arccos \left(\frac{(\beta-1) \omega}{K\|\mathbf{v}\|}\right)
$$

Proof. First suppose $M_{\text {sync }}(\phi)$ is invariant. Then we look for $\phi$ values satisfying such condition on $M_{\text {sync }}(\phi)$. If the dynamics (5.2) is evaluated on $M_{\text {sync }}$ yields

$$
\dot{\phi}=(1-\beta) \omega-K \sin (\phi-v)-\beta K \sin (\phi+v) .
$$

In order to be invariant the additional fact is $\dot{\phi}=0$. The phase $\phi$ satisfying such synchronous condition will be denoted by $\zeta$ and holds the following:

$$
\sin (\zeta-v)+\beta \sin (\zeta+v)=\frac{(1-\beta) \omega}{K}
$$

Let us rearrange slightly the left-hand side of above condition

$$
\begin{aligned}
\sin (\zeta-v)+\beta \sin (\zeta+v) & =\sin (\zeta-v)+\beta \sin (\zeta-v+2 v) \\
& =(1+\beta \cos (2 v)) \sin (\zeta-v)+\beta \sin (2 v) \cos (\zeta-v) \\
& =\langle\mathbf{v},(\cos (\zeta-v), \sin (\zeta-v))\rangle,
\end{aligned}
$$

where $\mathbf{v}=(\beta \sin (2 v), 1+\beta \cos (2 v))$. Thus Equation (5.7) is equal to

$$
\langle\mathbf{v},(\cos (\zeta-v), \sin (\zeta-v))\rangle=\frac{(1-\beta) \omega}{K}
$$


The inner product is induced by the metric on $\mathbb{R}^{2}$, so clearly it must satisfy $\|\mathbf{v}\| \geq$ $\frac{|1-\beta| \omega}{K}$. Such condition gives us the backward critical coupling

$$
K_{c}^{b}:=\frac{(\beta-1) \omega}{\|\mathbf{v}\|}
$$

without the modulus on $\beta-1$ since $\beta>1$. The stability of $M_{\text {sync }}$ is analyzed considering the lineazired vector field. The system (5.2) can be written as: $\dot{\Phi}=F_{\beta, \omega, K, v}(\Phi)$, where $\Phi=\left(\phi_{1}, \ldots, \phi_{N}, \vartheta_{H}\right) \in \mathbb{T}^{N+1}$ and the vector field $F_{\beta, \omega, K, v}: \mathbb{T}^{N+1} \rightarrow \mathbb{T}^{N+1}$ with

$$
F_{\beta, \omega, K, v}(\Phi)=\left(\dot{\phi}_{1}, \ldots, \dot{\phi}_{N}, \dot{\vartheta}_{H}\right)
$$

where $\beta, \omega, K, v$ are parameters which define a family of vector fields. We select a vector field fixing parameter's values. The total derivative evaluated at coherent state is

$D F_{\beta, \omega, K, v}(\zeta)=\left(\begin{array}{cccc}-K \cos (\zeta-v)-\beta K \cos (\zeta+v) & \cdots & 0 & 0 \\ \vdots & \ddots & \vdots & 0 \\ 0 & \cdots & -K \cos (\zeta-v)-\beta K \cos (\zeta+v) & 0 \\ \frac{\beta K}{N} \cos (\zeta+v) & \cdots & \frac{\beta K}{N} \cos (\zeta+v) & 0\end{array}\right)$.

Rewriting we obtain

$$
D F_{\beta, \omega, K, v}(\zeta)=-K \cos (\zeta-v) \operatorname{Diag}(1,1, \ldots, 0)+\beta K \cos (\zeta+v)\left(\begin{array}{cccc}
-1 & \cdots & 0 & 0 \\
\vdots & \ddots & \vdots & 0 \\
0 & \cdots & -1 & 0 \\
\frac{1}{N} & \cdots & \frac{1}{N} & 0
\end{array}\right) .
$$

The matrix has a block diagonal form, then the eigenvalues are obtained effortless

$$
\lambda_{1}=0 \text { and } \lambda_{2}=-K \cos (\zeta-v)-\beta K \cos (\zeta+v),
$$

where there is one eigenvector $(0, \ldots, 0,1)$ associated to $\lambda_{1}$ and $N$ independent eigenvectors of the form $(0, \ldots, 1,0,-1, \ldots, 0)$ associated to $\lambda_{2}$ which has algebraic multiplicity $N$. The first eigenvector indicates that along $M_{\text {sync }}$ the tangent flow is neutrally stable since $\lambda_{1}$ is zero. 


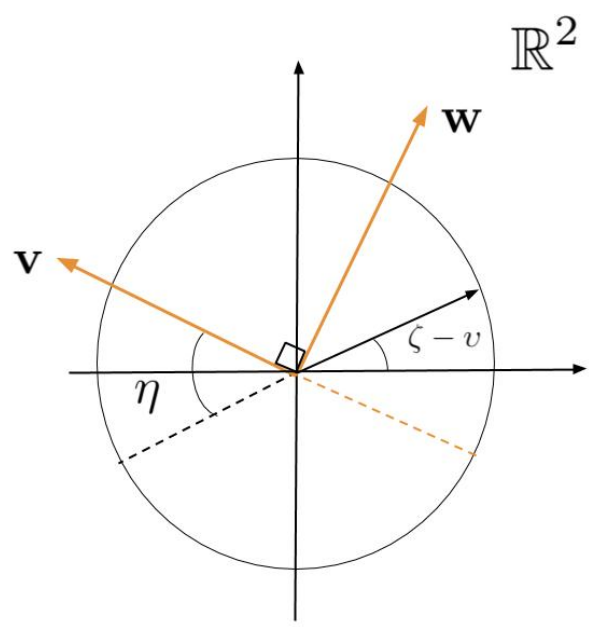

Figure 8 - Geometrical interpretation of vectors $\mathbf{v}$ and $\mathbf{w}$. The fixed point $\zeta-v$ is arbitrary shown as a unit vector. Since the intern product between $\mathbf{v}$ and unit vector with $\zeta-v$ is negative, they are more the $\pi / 2$ apart.

Source: By the author.

On the other hand, transversally we must use the assumption on $M_{\text {sync }}$ to be normally attracting. So, $\lambda_{2}<0$ lead us to a new condition for the parameters:

$$
K \cos (\zeta-v)+\beta K \cos (\zeta+v)>0 .
$$

Similar to the first condition, Equation (5.14) is rearranged considering $K>0$ and results

$$
\langle\mathbf{w},(\cos (\zeta-v), \sin (\zeta-v))\rangle>0
$$

where $\mathbf{w}=(1+\beta \cos (2 v),-\beta \sin (2 v)) \perp \mathbf{v}$. These two conditions 5.7 and 5.14 must be satisfied in order to $M_{\text {sync }}$ be normally attracting. This request a geometric interpretation in order to find the $\zeta$ value.

Visualizing the conditions 5.7 and 5.14 geometrically, see Figure 8. Such interpretation lead us to $\zeta$ can be obtained from the sum of angles in the figure and thus, we must use the Hypothesis 5.0.2. We must calculate the angle $\eta$ between $\mathbf{v}$ and unit vector $(\cos (\zeta-v), \sin (\zeta-v)) \in \mathbb{R}^{2}$. Since $\mathbf{w} \perp \mathbf{v}$ and (5.15) must hold: $\eta>0$. It turns out that $\|\mathbf{v}\|>\frac{|1-\beta| \omega}{K}$ is a strictly inequality, where $K_{c}^{b}=\frac{|1-\beta| \omega}{\|\mathbf{v}\|}$.

The inner product between the vectors $(5.8)$ is always negative $(\beta>1)$, then we calculate $\zeta-v$ rotated by $\pi$ 


$$
\eta=\arccos \left(\frac{(\beta-1) \omega}{K\|\mathbf{v}\|}\right)
$$

where $\eta \in(-\pi / 2, \pi / 2)$. With this in hand, finally the expression of phase $\zeta$ is

$$
\zeta=v-\pi+\arg (\mathbf{v})+\eta
$$

where $\arg (\mathbf{v})=\arctan \left(\frac{1+\beta \cos (2 v)}{\beta \sin (2 v)}\right)$. This proves one direction. The converse is straightforward.

\subsubsection{Asynchronous manifold}

With the intention to use 4.1.1, Equation 5.2 is written in an proper form. After some algebraic manipulations we find

$$
\begin{array}{ll}
f=-\frac{K e^{-i v}}{2 i} & g=(1-\beta) \omega-\beta \frac{K}{N} \sum_{j=1}^{N} \sin \left(\phi_{j}+v\right), \\
f=-\frac{K e^{-i v}}{2 i} & g=(1-\beta) \omega-\beta \frac{K}{2 i} e^{i v} r+\beta \frac{K}{2 i} e^{-i v} \bar{r},
\end{array}
$$

which fits the form (4.1). Thus, system (5.1) belongs to the large class of oscillators required by Theorem 4.1.1. Equations for $\alpha$ and $\psi$ are obtained if we replace $f$ and $g$ by the above expressions. However these expressions are tricky, once involve the mapping $\pi$ of Equation (4.3) for each $\phi_{j}$ in the sum argument. We skip the step of writing down the equations for them since we are not able to solve them.

From Lemma 4.2.1, $\alpha$ is close to order parameter $r$ when we consider $\mathbb{D}_{\delta} \times S^{1} \times U$. Thus, we adopt $r=\alpha$ in Equation (5.18), closing equation for $\alpha$, and consequently, decoupling from $\psi$. It yields

$$
\dot{\alpha}=-\frac{K}{2}\left(e^{-i v}+\beta e^{i v}\right) \alpha^{2}+i(1-\beta) \omega \alpha+\frac{K}{2}\left(\beta e^{-i v}|\alpha|^{2}+e^{i v}\right)
$$

with $v>0$. The next proposition characterizes the dynamics of the asynchronous state.

Proposition 5.0.4. Let the Equation (5.19) with $\beta>1$ and $\omega>0$. If $0<v<\pi / 4$ then for each 


$$
0<K<K_{c}^{f}:=\frac{(\beta-1) \omega}{\sqrt{1+2 \beta \cos 2 v}}
$$

there exists a family of hyperbolic incoherent states

$$
\left|\alpha_{\text {async }}^{-}(K, \beta, v)\right|=\frac{(\beta-1) \omega-\sqrt{(\beta-1)^{2} \omega^{2}-K^{2}(1+2 \beta \cos 2 v)}}{K(1+2 \beta \cos 2 v)} .
$$

and for each

$$
\frac{(\beta-1)}{(1+\beta \cos (2 v))}<K<K_{c}^{f}
$$

there exists a family of hyperbolic incoherent states

$$
\left|\alpha_{\text {async }}^{+}(K, \beta, v)\right|=\frac{(\beta-1) \omega+\sqrt{(\beta-1)^{2} \omega^{2}-K^{2}(1+2 \beta \cos 2 v)}}{K(1+2 \beta \cos 2 v)} .
$$

Consequently, $\left|\alpha_{\text {async }}^{-}\right|$is the unique branch of stationary solution when $K$ is close to zero.

Proof. The complex variable $\alpha$ is written in polar coordinates as $r \in(0, \infty)$ and $\chi \in S^{1}$ : $\alpha=\rho e^{i \chi}$. Replacing it in Equation (5.19) yields

$$
\begin{aligned}
& \dot{\rho}=\frac{K}{2}\left(1-\rho^{2}\right) \cos (\chi-v) \\
& \dot{\chi}=-K \beta \rho \sin (\chi+v)+\frac{K}{2} \frac{\rho^{2}+1}{\rho} \sin (v-\chi)+(1-\beta) \omega .
\end{aligned}
$$

The stationary state is the fixed points of above system (5.23). We set $\dot{\rho}=\rho \dot{\chi}=0$, where $\rho \neq 0$ when $K>0$. From equation for $\rho$, we determine components of three fixed points $\rho_{*}=1$ and $\chi_{*}^{ \pm}= \pm \pi / 2+v$.

The fixed point with radius component $\rho_{*}$ is not valid since is out of the domain $\mathbb{D}_{\delta}$. The angle with positive sign $\chi_{*}^{+}$yields unreal results for radial component, regarding the condition for $\beta \in(1, \infty)$. Then, the unique accessible fixed point is with angular component with negative sign. We replace such angle $\chi_{*}^{-}=-\pi / 2+v$ in (5.23) in order to determine the radial component

$$
\rho_{*}^{ \pm}=\frac{(\beta-1) \omega \pm \sqrt{(\beta-1)^{2} \omega^{2}-K^{2}(1+2 \beta \cos (2 v))}}{K(1+2 \beta \cos (2 v))} .
$$


The radial component is a real variable, then the coupling must satisfy

$$
K<K_{c}^{f}:=\frac{(\beta-1) \omega}{\sqrt{1+2 \beta \cos (2 v)}}
$$

where $K_{c}^{f}$ is called the forward critical coupling. Besides, the radial component is valid inside a domain, where $\alpha \in \mathbb{D}_{\delta}$. Thus, any of the branches must have $\rho_{*}^{ \pm}<1$. Consider the following

$$
\begin{aligned}
\left|\frac{(\beta-1) \omega \pm \sqrt{(\beta-1)^{2} \omega^{2}-K^{2}(1+2 \beta \cos (2 v))}}{K(1+2 \beta \cos (2 v))}\right| & <1 \\
\pm \sqrt{(\beta-1)^{2} \omega^{2}-K^{2}(1+2 \beta \cos (2 v))} & <K(1+2 \beta \cos (2 v))-(\beta-1) \omega
\end{aligned}
$$

where we separate in two cases. The positive branch after some algebraic considerations exist if

$$
K>\frac{(\beta-1) \omega}{(1+\beta \cos (2 v))}
$$

On the other hand, the negative branch has no restriction. Consequently, we prove that the negative branch $\left|a_{\text {async }}^{-}\right|$is unique in the limit of $K \rightarrow 0$.

Regarding these couplings strength we determine a stability analysis of these two fixed points $\left(\rho_{*}^{ \pm}, \chi_{*}^{-}\right)$. Evaluating the Jacobian matrix of the Equation (5.23) at these fixed points we get

$$
D F\left(\rho_{*}^{ \pm}, \chi_{*}^{-}\right)=\left(\begin{array}{cc}
0 & \frac{K}{2}\left(1-\left(\rho_{*}^{ \pm}\right)^{2}\right) \\
K \beta \cos (2 v)+\frac{K}{2}\left(1-\frac{1}{\left(\rho_{*}^{ \pm}\right)^{2}}\right) & -\left(K \beta \rho_{*}^{ \pm} \sin (2 v)\right)
\end{array}\right) .
$$

Finding explicit expressions for the eigenvalues is useless, since for two dimensional linear systems the eigenvalues are written in terms of trace and determinant of the Jacobian matrix. Thus the stability of fixed points considering parameters values follows the characterization below.

- For $\frac{(\beta-1)}{(1+\beta \cos (2 v))}<K<K_{c}^{f}:\left(\rho_{*}^{+}, \chi_{*}^{-}\right)$is a saddle fixed points since $\operatorname{Tr}\left(D F\left(\rho_{*}^{+}, \chi_{*}^{-}\right)\right)<$ 0 and $\operatorname{det}\left(D F\left(\rho_{*}^{+}, \chi_{*}^{-}\right)\right)<0$.

- For $0<K<K_{c}^{f}$ : $\left(\rho_{*}^{-}, \chi_{*}^{-}\right)$is a sink fixed point since $\operatorname{Tr}\left(D F\left(\rho_{*}^{-}, \chi_{*}^{-}\right)\right)<0$ and $\operatorname{det}\left(D F\left(\rho_{*}^{-}, \chi_{*}^{-}\right)\right)>0$.

Both fixed points are hyperbolic, because the eigenvalues have real part different then zero. For this reason, the proof is complete. 
Remark 5.0.5. Inside the interval considered $\beta \in(1, \infty)$ is always true that $K_{c}^{b}<K_{c}^{f}$. In addition, if $\beta \in(0,1)$ the backward critical couplings is negative. Then, for $K>0$ the synchronous manifold is always attracting. Further investigations are necessary to verify conditions on asynchronous branches.

Remark 5.0.6 (Separatrix). The saddle node $\left(\rho_{*}^{+}, \chi_{*}^{-}\right)$found is accessed depending on the initial conditions. Regarding the space $\mathbb{D}_{\delta} \times S^{1}$, we say the invariant manifolds associated to such saddle node have measure zero. So, for generic initial conditions the system never reaches such macroscopic state, always going to totally synchronous or asynchronous state. The interval of existence of this branch we say is the area of coexistence of coherent and incoherent states.

The above remark indicates that we can drop the notation of \pm to denote asynchronous branches. From here on we denote simply by $\alpha_{\text {async }}=\rho_{*}^{+} e^{i \chi_{*}^{+}}$the accessible positive branch for generic initial conditions.

The previous characterization determined the asynchrounous state in terms of $\alpha$. However, our goal is determining in terms of the order parameter $r$. Then we state proposition 5.0.7 where the asynchronous manifold is finally defined on $\mathbb{T}^{N}$.

Proposition 5.0.7. Let $0<K<K_{c}^{f}$ and $0<v<\pi / 4$. Given $\varepsilon>0$ there exist $N_{0}$ such that for each $N>N_{0}$ there exists a normally hyperbolic invariant manifold $M_{\text {async }} \subset \mathbb{T}^{N}$ such that

$$
\left\|r-\alpha_{\text {async }}\right\|_{C^{k}} \leq \varepsilon
$$

where $\left|\alpha_{\text {async }}\right|$ is given by Equation (5.20).

Proof. Consider the system given by Equation (5.1). From Möbius group reduction, one changes coordinates to $\left(\alpha, \psi, \theta_{1}, \ldots, \theta_{N}\right)$ where follow the Equation (4.4). If we replace the condition of splay state in $f$ and $g$ expression, one obtains that $\alpha$ equation decouples from $\psi$ equation and $\theta$ 's are considered fixed parameters. With this in hand, we find a value $\alpha_{\text {async }} \in \mathbb{D}$ given by (5.20) that is linearly stable fixed point. The vector field of $\psi$ equation only depends on $\alpha$ and linearly has zero eigenvalue (is neutral). It follows that $M_{\alpha, \psi}=\left\{(\alpha, \psi) \subset \mathbb{D} \times S^{1} \mid \alpha=\alpha_{\text {async }}\right\}$ is two-dimensional submanifold of $\mathbb{D} \times S^{1}$ and is normally hyperbolic invariant manifold.

From Lemma 4.2.1, given $k \geq 1$ and a ball $B_{\delta}\left(\alpha_{\text {async }}\right) \in \mathbb{D}$, there exists an $N$ and a neighborhood $U \subset \mathbb{T}^{N}$ of the splay state $\Theta$ such that $r\left(\alpha, \psi, \theta_{1}, \ldots, \theta_{N}\right)$ replaced by $\alpha$ is a $C^{k}$-small perturbation of the system (4.4). Therefore, by persistence of normally hyperbolic invariant manifolds theorem 3.0.11, the dynamics of $r$ also has a normally hyperbolic invariant manifold 


$$
\tilde{M}_{\alpha, \psi}(\Theta)=\left\{\alpha=\alpha_{\text {async }}+g_{\text {async }}(\psi, \Theta)\right\} \subset \mathbb{D} \times S^{1}
$$

close to $M_{\alpha, \psi}$. That is, uniformly for all $\Theta \in U$ we have $\left\|g_{\text {async }}\right\|_{C^{k}} \ll 1$ and $\|r-\alpha\|_{C^{k}} \ll 1$ on $\tilde{M}_{\alpha, \psi}(\Theta)$ also. From here on let $\Theta \in T^{N}$ as dynamical variables again. Since the $\theta_{i}$ 's have no dynamic, also

$$
M_{\text {async }}=\bigcup_{\Theta \in U} \tilde{M}_{\alpha, \psi}(\Theta) \subset \mathbb{D} \times S^{1} \times \mathbb{T}^{N}
$$

is $N$-dimensional normally hyperbolic invariant manifold and defines the asynchronous manifold.

Finally the last step follows from a straightforward calculation. Let the negative branch of Proposition 5.0.4. Given a $\sigma>0$ take expression of $\left|\alpha_{\text {async }}^{-}\right|$in Equation (5.20). We estimate the magnitude of $\left|\alpha_{\text {async }}^{-}\right|$at $K_{c}^{f}$ which is the largest value regarding this branch existence:

$$
\begin{aligned}
\left|\alpha_{\text {async }}^{-}\right| & =\left|\frac{1}{\sqrt{1+2 \beta \cos (2 v)}}\right| \\
& <\left|\frac{1}{\sqrt{1+2 \beta_{0} \cos (2 v)}}\right| \leq \sigma
\end{aligned}
$$

for a sufficient large $\beta_{0}$. With this, we complete the proof. 


\section{Conclusions}

The main purpose of this dissertation was to tackle transitions phenomena from incoherent towards coherent behavior in star motifs. We have proved in rigor that this transition is discontinuous when positive correlation setting is chosen. We pointed out that the key feature in the discontinuity is the correlation parameter $\beta$ inside the interval $(1, \infty)$. It affects existence and stability conditions of the collective dynamics measured by the order parameter. On one hand, the synchronous state is a fixed point for order parameter equation. On the other hand, to write down the incoherent dynamics in terms of the order parameter we needed to use: Möbius group reduction, splay-state hypothesis condition and persistence of normally attracting invariant manifolds.

We achieved the goal in revealing that explosive synchronization phenomenon is discontinuous in finite size star motifs. Moreover, this local description of collective behavior sheds light on dynamics of units coupled in more complex connection patterns. 



\section{References}

1 GÓMEZ-GARDEÑES, J. et al. Explosive synchronization transitions in scale-free networks. Physical Review Letters, v. 106, n. 12, p. 128701. doi: 10.1103/PhysRevLett.106.128701.

2 ZOU, Y. et al. Basin of attraction determines hysteresis in explosive synchronization. Physical Review Letters, v. 112, n. 11, p. 114102, 2014.

3 SKARDAL, P. S.; TAYLOR, D.; SUN, J. Optimal synchronization of complex networks. Physical Review Letters, v. 113, n. 14, p. 144101, 2014.

4 PERON, T. K. D.; RODRIGUES, F. A. Explosive synchronization enhanced by time-delayed coupling. Physical Review E, v. 86, n. 1, p. 016102, 2012.

5 PERON, T. K. D.; RODRIGUES, F. A. Determination of the critical coupling of explosive synchronization transitions in scale-free networks by mean-field approximations. Physical Review E, v. 86, n. 5, p. 056108, 2012.

6 COUTINHO, B. C. et al. Kuramoto model with frequency-degree correlations on complex networks. Physical Review E, v. 87, n. 3, p. 032106, 2013.

7 VLASOV, V.; ZOU, Y.; PEREIRA, T. Explosive synchronization is discontinuous. Physical Review E, v. 92, n. 1, p. 012904, 2015.

8 BARABÁSI, A.-L.; ALBERT, R. Emergence of scaling in random networks. Science, v. 286, n. 5439, p. 509-512, 1999.

9 PEREIRA, T. et al. Towards a theory for diffusive coupling functions allowing persistent synchronization. Nonlinearity, v. 27, n. 3, p. 501, 2014.

10 WARNER, F. W. Foundations of differentiable manifolds and Lie groups. New York: Springer, 1983.

11 CHICONE, C. Ordinary differential equations with applications. 2nd ed. New York: Springer, 2006. (Texts in applied mathematics, v.34).

12 TESCHL, G. Ordinary differential equations and dynamical systems. Providence: American Mathematical Society, 2004. (Graduate studies in mathematics; v.140).

13 HERSTEIN, I. N. Topics in algebra. 2nd ed. New York: Wiley, 1975.

14 OLVER, P. J. Classical invariant theory. Cambridge, UK: Cambridge University Press, 1999.

15 MARVEL, S. A.; MIROLlO, R. E.; STROGATZ, S. H. Identical phase oscillators with global sinusoidal coupling evolve by möbius group action. Chaos, v. 19, n. 4, p. 043104, 2009 .

16 StEIN, E. M.; SHAKARCHI, R. Complex analysis. Princeton, New Jersey:

Princeton University Press, 2003. (Princeton lectures in analysis, 2). 
17 HALL, B. Lie groups, Lie algebras, and representations: an elementary introduction. New York: Springer-Verlag, 2003.

18 IBRAGIMOV, N. K. Group analysis of ordinary differential equations and the invariance principle in mathematical physics (for the 150th anniversary of Sophus Lie). Russian Mathematical Surveys, v. 47, n. 4, p. 89, 1992.

19 STEWART, I. Phase oscillators with sinusoidal coupling interpreted in terms of projective geometry. International Journal of Bifurcation and Chaos, v. 21, n. 06, p. 1795-1804, 2011.

20 COPPEL, W. A. Dichotomies in stability theory. Berlin: Springer-Verlag, 1978. (Lectures notes in mathematics v. 629).

21 STANKOVSKI, T. et al. Coupling functions: Universal insights into dynamical interaction mechanisms. Review of Modern Physics, v. 89, n. 4, p. 045001, 2017.

22 LANCASTER, P.; TISMENETSKY, M. The theory of matrices: with applications. 2nd ed. San Diego: San Diego Academic Press, 1985.

23 KUZNETSOV, Y. A. Elements of applied bifurcation theory. New York: Springer, 1998.

24 RUDIN, W. Principles of mathematical analysis. 3rd ed. New York: McGraw-Hill, 1976.

25 WIGGINS, S. Normally hyperbolic invariant manifolds in dynamical systems. New York: Springer-Verlag, 1994. (Applied mathematical sciences, v. 105).

26 FENICHEL, N. Persistence and smoothness of invariant manifolds for flows. Indiana University Mathematics Journal, v. 21, n. 3, p. 193-226, 1972.

27 PIKOVSKY, A.; ROSENBLUM, M. Partially integrable dynamics of hierarchical populations of coupled oscillators. Physical Review Letters, v. 101, p. 264103, 2008. 